\title{
Scaling of X-ray spectral properties of a black hole in the Seyfert 1 galaxy NGC 7469
}

\author{
Elena Seifina ${ }^{1,3}$, Lev Titarchuk ${ }^{2,3}$, and Lyubov Ugolkova ${ }^{3}$ \\ ${ }^{1}$ LAPTh, Universite Savoie Mont Blanc, CNRS, BP 110, 74941 Annecy-le-Vieux, France \\ e-mail: seifina@lapth.cnrs.fr \\ 2 Dipartimento di Fisica, Università di Ferrara, Via Saragat 1, 44122 Ferrara, Italy \\ e-mail: titarchuk@fe.infn.it \\ 3 Moscow State University/Sternberg Astronomical Institute, Universitetsky Prospect 13, Moscow 119992, Russia \\ e-mail: seif@sai.msu.ru
}

Received 5 April 2018 / Accepted 22 July 2018

\begin{abstract}
We present our analysis of X-ray spectral properties observed from the Seyfert 1 galactic nucleus NGC 7469 using the Rossi X-ray Timing Explorer (RXTE) and Advanced Satellite for Cosmology and Astrophysics mission (ASCA) observations. We demonstrate strong observational evidence that NGC 7469 undergoes spectral transitions from the low hard state (LHS) to the intermediate state (IS) during these observations. The RXTE observations (1996-2009) show that the source was in the IS 75\% of the time only 25\% of the time in the LHS. The spectra of NGC 7469 are well fitted by the so-called bulk motion Comptonization (BMC) model for all spectral states. We have established the photon index $(\Gamma)$ saturation level, $\Gamma_{\text {sat }}=2.1 \pm 0.1$, in the $\Gamma$ versus mass accretion rate, $\dot{M}$ correlation. This $\Gamma-\dot{M}$ correlation allows us to estimate the black hole (BH) mass in NGC 7469 to be $M_{\mathrm{BH}} \geq 3 \times 10^{6} M_{\odot}$ assuming the distance to NGC 7469 of 70 Mpc. For this BH mass estimate, we use the scaling method taking Galactic BHs, GRO J1655-40, Cyg X-1, and an extragalactic BH source, NGC 4051 as reference sources. The $\Gamma$ versus $\dot{M}$ correlation revealed in NGC 7469 is similar to those in a number of Galactic and extragalactic BHs and it clearly shows the correlation along with the strong $\Gamma$ saturation at $\approx 2.1$. This is robust observational evidence for the presence of a BH in NGC 7469. We also find that the seed (disk) photon temperatures are quite low, of the order of $140-200 \mathrm{eV}$, which are consistent with a high BH mass in NGC 7469 that is more than $3 \times 10^{6}$ solar masses.
\end{abstract}

Key words. accretion, accretion disks - black hole physics - galaxies: active - galaxies: individual: NGC 7469 - galaxies: Seyfert

\section{Introduction}

Active galactic nuclei (AGNs) belong to the most luminous objects in the Universe, frequently outshining their host galaxies. Historically, AGN sources have been classified using their optical and radio characteristics and were initially considered as a complex of different objects. It is now thought that all AGNs are the same in terms of a common central engine, namely, an accreting supermassive black hole (SMBH) located at the center of its host galaxy. Several of the observed differences in these sources can be explained by angle-dependence and obscuration effects. However, these properties cannot be enough to explain the great variety of AGN properties that have been discovered. It is very desirable to study AGNs in X-rays in combination with optical and radio emission using spectral and timing analysis.

Among such AGNs, NGC 7469 is a famous Seyfert 1 galaxy located in the Pegasus constellation. In optical emission this source was discovered more than seventy years ago by Carl Seyfert (Seyfert 1943). X-rays from NGC 7469 were first detected by Uhuru (Forman 1978). The main feature of this galaxy is the activity of its central region detected in optical and X-rays as a compact, variable source, and the presence of an ultraviolet emission excess from the galaxy's central region. The galaxy NGC 7469 (Arp $298=$ MCG 1-58-25 = Mrk 1514) is classified as a spiral SBa galaxy slightly inclined to the line of sight. The distance to NGC 7469 is estimated to be in a wide range (from 50 to $117 \mathrm{Mpc}$ ) obtained by different methods: the
AGN time lag (47-117 Mpc; Peterson et al. 2004, 2014), SN Ia (66 Mpc; Ganeshalingam et al. 2013), Tully-Fisher (40-61 Mpc; Bottinelli et al. 1984; Theureau et al. 2007), and others.

We take the rest frame of NGC 7469 to be at a redshift of $z=0.016268$, based on the $21 \mathrm{~cm}$ line (Spirandob et al. 2005; Peterson et al. 2004), and thus luminosity distance to be $70 \mathrm{Mpc}$. Analyses of X-ray Chandra and XMM-Newton grating spectra of NGC 7469 (Scott et al. 2005) provide the total absorbing column density in the X-rays of order $3 \times 10^{21} \mathrm{~cm}^{-2}$. For the neutral absorption in our Galaxy, we use a column density of $6 \times 10^{20} \mathrm{~cm}^{-2}$ that includes both $\mathrm{H} 1\left(4.34 \times 10^{20} \mathrm{~cm}^{-2}\right)$ and $\mathrm{H}_{2}\left(5.75 \times 10^{19} \mathrm{~cm}^{-2}\right.$, Wakker et al. 2011), for a total of $\sim 3 \times 10^{21} \mathrm{~cm}^{-2}$ (Behar et al. 2017). Around the central part of this galaxy, two ring areas of star formation are observed. The central region of NGC 7469 exhibits variability in the $\mathrm{X}$-ray, ultraviolet, optical, and infrared bands as well as in spectral lines. However, NGC 7469 is a weak radio source as are most of all known Seyfert galaxies.

NGC 7469 is well studied in optical emission (see, e.g., Doroshenko et al. 1989; Merkulova 2000; Sergeev et al. 2005) using the facilities of the Crimean Astrophysical Observatory and the Crimean Laboratory of the Sternberg Astronomical Institute ( $U, B, V$ light curves are presented in Fig. 1). It is worth noting that the AGN Watch international project organized in the mid 1990s (see Alloin et al. 1994) obtained numerous results on the optical variabilities of NGC 7469 (see Netzer \& Peterson 1997; Ulrich et al. 1997; O’Brien \& Leighly 1998) and on 
ultra-rapid variability (Dultzin-Hacyan et al. 1992, 1993). Results of the Hubble Space Telescope and the Multicolor active Galactic Nuclei Monitoring (MAGNUM) telescope (2001-2003) observations of NGC 7469 are presented in Suganuma et al. (2006). Analysis of MAGNUM light curves indicates the presence of short-term variability, from several days to several weeks. Optical observations of NGC 7469, particularly observations performed in the Maidanak observatory (Artamonov et al. 2010), indicate long-term (eight-nine years) variability of the nucleus with outbursts of a relatively long rise (one-two years), peak ( one-two years), and long decay (three years).

Several giant outbursts in NGC 7469 are known to have been observed in UBVRI bands (see bottom panels in Fig. 1) with maxima in 1993 (Lyutyi et al. 1995; Lyuty 2005; Doroshenko et al. 1998), 1997-1998, 2005 (Doroshenko et al. 2010; Artamonov et al. 2010; Ugolkova \& Artamonov 2011), and 2012 (Ugolkova et al. 2017; Shapovalova \& Popovic 2017). Unfortunately, $\mathrm{X}$-ray pointed observations with the Rossi X-ray Timing Explorer (RXTE) cover only partial phases: a rise (1996) of the 19942001 outburst, a decay in 2003, and a rise in 2009 (see Ugolkova et al. 2017), while soft X-ray monitoring observations (ASM, 2$10 \mathrm{keV})^{1}$ cover continuously the whole (1996-2011) interval (see top panel in Fig. 1). It is worth noting that the soft X-ray emission from NGC 7469 demonstrates a more complex pattern than that in the optical band (compare top and bottom panels in Fig. 1). Generally, the source shows numerous short-term X-ray outbursts (on the order of a few days) and sparse long-term optical outbursts (six-eight years), modified by rapid variability (for more details see Sect. 3.1). A stellar disruption was suggested as an explanation for the great outburst that occurred in 1997-1998 (Rees 1988). In the present paper we reanalyzed X-ray observations and matched them with optical ones in order to diagnose the source activity states based on X-ray/optical light curve pattern.

It is important to emphasize that the optical-X-ray connections observed in NGC 7469 are very different for various epoches. To explain these differences, Gaskell (2006, 2007) suggested a model using X-ray anisotropy in combination with an effect of the dust surrounding the accretion disk, which can cause the lags between variations in X-ray and optical bands. The physics of processes near the nucleus of NGC 7469 are also discussed in detail by Doroshenko et al. (2010), Chesnok et al. (2009), Ugolkova et al. (2017). Using a comparison of the X-ray and optical properties of NGC 7469, these authors concluded that the direct Compton X-ray reprocessing cannot provide the whole observed optical luminosity of NGC 7469. Other processes, such as thermal radiation from the accretion disk, the star formation processes, and inverse Compton scattering by hot coronal electrons may also contribute to the optical emission.

The mass of the SMBH centered in NGC 7469 is estimated over a wide range of $M=(1-6) \times 10^{7} M_{\odot}$ (Peterson et al. 2004, 2014; Shapovalova \& Popovic 2017) by using the reverberation mapping method. It is desirable to use an independent black hole $(\mathrm{BH})$ identification for its central object as well as the mass estimate of its $\mathrm{BH}$ by an alternative to the abovementioned method. A method of $\mathrm{BH}$ mass determination was developed by Shaposhnikov \& Titarchuk (2009), hereafter ST09, using a correlation scaling between X-ray spectral and timing (or mass accretion rate) properties observed from many Galactic BH binaries during $\mathrm{BH}$ state transitions.

We apply the ST09 method to the Advanced Satellite for Cosmology and Astrophysics mission (ASCA) and RXTE data of NGC 7469. Previously, many X-ray properties of NGC 7469

HEASARC, http://xte.mit.edu/ASM_lc.html were analyzed using the European X-ray Observatory Satellite (EXOSAT), GINGA, and ASCA data, which enabled the detection and study of the soft excess of source X-ray spectra (Barr 1986; Turner et al. 1991, 1993). The GINGA observations revealed evidence for $6.4 \mathrm{keV}$ iron florescence lines and flattening of the spectral shape above $10 \mathrm{keV}$ (Piro et al. 1990). Spectral fits using a Comptonization model Matt et al. (1991), George \& Fabian (1991) yielded a power-law photon index of $\Gamma \sim 2$ (Piro et al. 1990; Nandra et al. 1991). Scott et al. (2005) investigated simultaneous X-ray, far-ultraviolet, and near-ultraviolet spectra of NGC 7469 using the Chandra X-Ray Observatory, the Far Ultraviolet Spectroscopic Explorer, and the Space Telescope Imaging Spectrograph on the Hubble Space Telescope. In particular, they detected $\mathrm{O}$ VIII emission as well as a prominent $\mathrm{Fe}$ $\mathrm{K}_{\alpha}$ emission line and absorption due to $\mathrm{H}$-like and He-like neon, and magnesium in the Chandra spectrum

In this paper we present an analysis of the available ASCA and RXTE observations of NGC 7469 to reexamine previous conclusions on the $\mathrm{BH}$ nature of NGC 7469 as well as to find further indications of a supermassive BH in NGC 7469. In Sect. 2 we present the list of observations used in our data analysis while in Sect. 3 we provide details of the X-ray spectral analysis. We discuss an evolution of the X-ray spectral properties during the spectral state transitions and present the results of the scaling analysis to estimate a BH mass for NGC 7469 in Sect. 4. We make our final conclusions on the results in Sect. 5 .

\section{Observations and data reduction}

Along with the long-term RXTE observations in 1996, 2003, 2005-2006, and 2009, which we describe in Sect. 2.2, NGC 7469 was observed by ASCA (1993, 1994, see Sect. 2.1). We extracted these data from the High Energy Astrophysics Science Archive Research Center (HEASARC) archives and found that these data cover a wide range of X-ray luminosities. We should also recognize that the well-exposed ASCA data are preferable for the determination of low-energy photoelectric absorption.

\subsection{ASCA data}

ASCA observed NGC 7469 on November 24-26, 1993. Table 1 summarizes the start time, end time, and the MJD interval for each of these observations. One can see a description of the ASCA experiment in Tanaka et al. (1994). The solid imaging spectrometers (SIS) operated in Faint CCD-2 mode. The ASCA data were screened using the ftool ascascreen and the standard screening criteria. The spectrum for the source was extracted using spatial regions with a diameter of 3' (for SolidState imaging Spectrometer (SISs) and 4' (for Gas Imaging spectrometer (GIS) centered on the nominal position of NGC 7469 $\left(\alpha=23^{\mathrm{h}} 03^{\mathrm{m}} 15^{\mathrm{s}} 75, \delta=+08^{\circ} 52^{\prime} 25^{\prime \prime} \cdot 9\right.$, J2000.0), while background was extracted from source-free regions of comparable size away from the source. The spectrum data were rebinned to provide at least 20 counts per spectral bin to validate the use of the $\chi^{2}$-statistic. The SIS and GIS data were fitted using XSPEC in the energy ranges of $0.6-10 \mathrm{keV}$ and $0.8-10 \mathrm{keV}$, where the spectral responses are best known.

The source count rate was variable by a factor of $40 \%$. We fit the spectral data using a number of models (see Sect. 3.2.1) but the best-fitting is obtained with the sum of XSPEC BMC (see Titarchuk et al. 1997) and Gaussian models while taking into account absorption at the low energies by neutral gas with solar composition. Using this model the amplitude of X-ray flux 


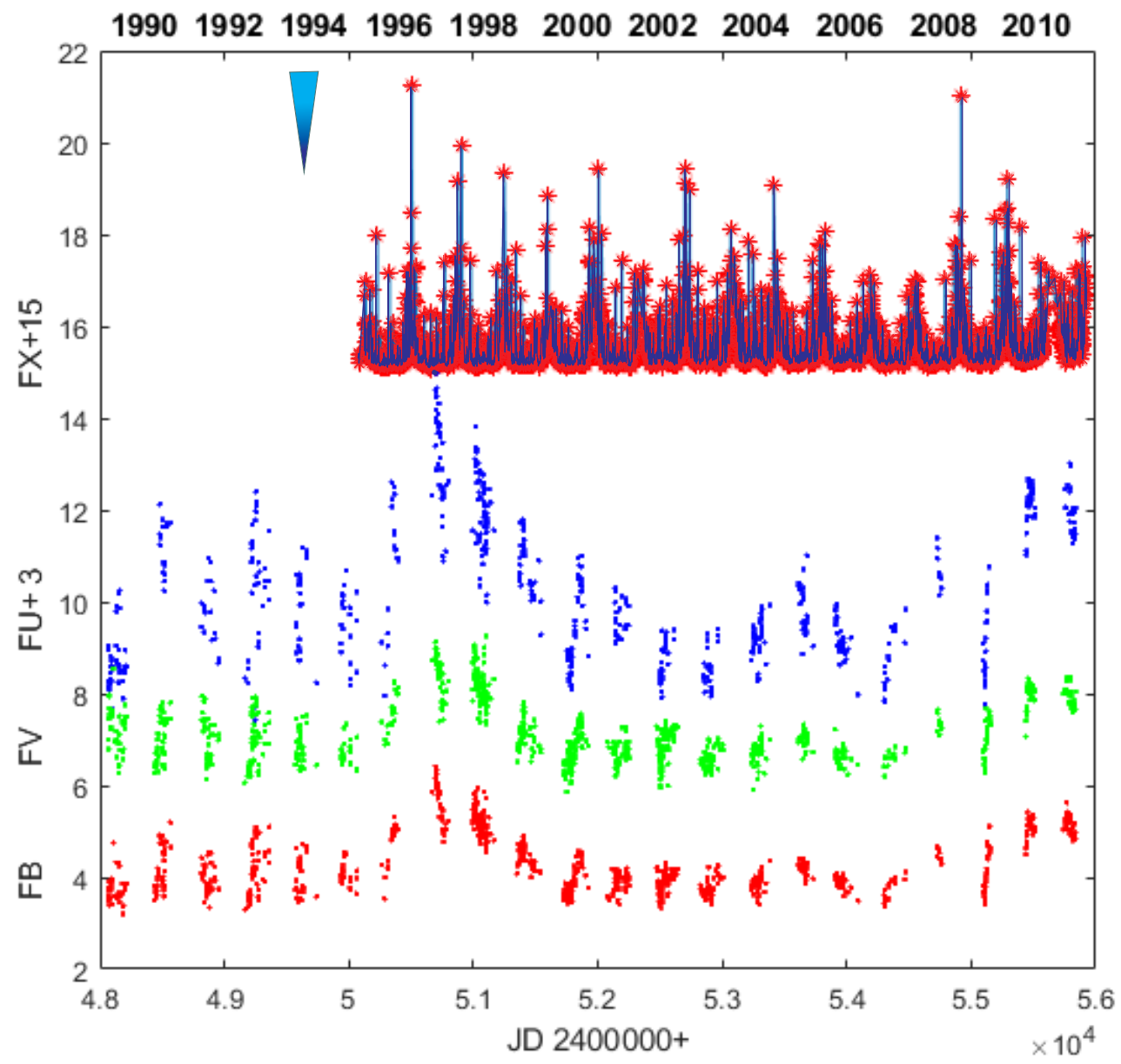

Fig. 1. X-ray light curve of NGC 7469 in the 2-12 keV energy range (1996-2011) is presented on the top. The bright blue triangle (arrow) indicates the ASCA observation MJD. The rate axis for the upper light curve (red stars) is related to the RXTE/ASM count rate increased by 15 units for clarity. In the three bottom panels the optical light curves (in $10^{-15} \mathrm{erg} \mathrm{cm}^{-2} \mathrm{~s}^{-1} \AA^{-1}$ ) of NGC 7469 in the $U$ (blue), $V$ (green), and $B$ (red) filters during 1995-2012 are presented. For clarity, the flux for the $U$-filter is increased by three units.

Table 1. ASCA observations of NGC 7469 used in our analysis.

\begin{tabular}{llllc}
\hline \hline Number of set & Obs. ID & Start time (UT) & End time (UT) & MJD interval \\
\hline A1 & 71028000 & 1993 Nov 24 05:28:38 & 1993 Nov 26 07:12:04 & $49315.2-49317.3^{1}$ \\
A2 & 71028010 & 1993 Dec 2 05:48:22 & 1993 Dec 3 01:22:04 & 49323.3-49324.1 \\
A3 & 71028030 & 1993 Nov 26 09:21:04 & 1993 Nov 26 19:23:14 & $49317.5-49317.8$ \\
A4 & 15030000 & 1994 June 26 23:02:40 & 1994 June 27 10:10:18 & 49529.9-49530.9 \\
A5 & 18931126 & 1993 Nov 26 07:39:12 & 1993 Nov 26 09:13:22 & 49317.3-49317.4 \\
\hline
\end{tabular}

References. (1) Guinazzi et al. (1994).

variability is up to a factor of two. The results of the fits are given in Table 2.

\subsection{RXTE data}

We also analyzed 263 RXTE observations made between April 1996 and December 2009 using the public archive. Standard tasks of the LHEASOFT/FTOOLS 5.3 software package were used for data processing. For spectral analysis we used PCA Standard 2 mode data, collected in the $3-23 \mathrm{keV}$ energy range, applying Proportional Conter Array (PCA) response calibration (ftool pcarmf v11.7). The fitting was carried out applying the standard XSPEC v 12.6.0 fitting package. The standard dead time correction procedure was applied to the data. The data from HEXTE detectors have been also used in order to construct the broad-band spectra. The spectral analysis of the data in the 19-50 keV energy range was implemented to account for the uncertainties in the HEXTE response and background determination. We subtracted a background corrected in off-source observations. The data are available through the Goddard Space Flifgt Center (GSFC) public archive ${ }^{2}$. We modeled the RXTE spectra using XSPEC astrophysical fitting software and we implemented a systematic uncertainty of $0.5 \%$ to all analyzed spectra. In Table 3 we listed the groups of RXTE observations tracing through the source evolution during different states.

\footnotetext{
2 http://heasarc.gsfc.nasa.gov
} 
Table 2. Best-fit parameters of the ASCA spectra of NGC 7469 in the $0.45-10 \mathrm{keV}$ range using the following five models: phabs*power, phabs*bbody, phabs*(bbody+power), phabs*bmc, and phabs*(bmc+Gaussian).

\begin{tabular}{|c|c|c|c|c|c|c|}
\hline Model & Parameter & 71028000 & 71028010 & 71028030 & 15030000 & 18931126 \\
\hline phabs & $N_{\mathrm{H}}\left(\times 10^{21} \mathrm{~cm}^{-2}\right)$ & $2.7 \pm 0.5$ & $2.5 \pm 0.9$ & $1.8 \pm 0.09$ & $2.6 \pm 0.2$ & $2.9 \pm 0.6$ \\
\hline \multirow[t]{3}{*}{ Power law } & $\Gamma_{\text {pow }}$ & $2.34 \pm 0.02$ & $2.29 \pm 0.02$ & $2.28 \pm 0.03$ & $2.31 \pm 0.02$ & $2.21 \pm 0.04$ \\
\hline & $N_{\text {pow }}^{\dagger \dagger}$ & $0.019 \pm 0.003$ & $0.019 \pm 0.001$ & $0.015 \pm 0.001$ & $0.036 \pm 0.001$ & $0.017 \pm 0.001$ \\
\hline & $\chi^{2}$ (d.o.f.) & $2.17(214)$ & $2.55(335)$ & $1.85(183)$ & $1.56(129)$ & $0.92(161)$ \\
\hline phabs & $N_{\mathrm{H}}\left(\times 10^{21} \mathrm{~cm}^{-2}\right)$ & $3.7 \pm 0.2$ & $3.5 \pm 0.3$ & $4.7 \pm 0.5$ & $5.8 \pm 0.3$ & $2.9 \pm 0.08$ \\
\hline \multirow{3}{*}{ Blackbody } & $T_{\mathrm{BB}}(\mathrm{keV})$ & $0.54 \pm 0.01$ & $0.36 \pm 0.01$ & $0.95 \pm 0.01$ & $0.89 \pm 0.01$ & $0.26 \pm 0.01$ \\
\hline & $N_{\mathrm{BB}}^{\dagger \dagger}$ & $5.8 \pm 0.4$ & $4.1 \pm 0.1$ & $12.3 \pm 0.4$ & $5.7 \pm 0.2$ & $27.9 \pm 0.7$ \\
\hline & $\chi^{2}$ (d.o.f.) & $8.61(214)$ & $14.1(335)$ & $2.63(183)$ & $4.08(129)$ & $15.76(161)$ \\
\hline phabs & $N_{\mathrm{H}}\left(\times 10^{21} \mathrm{~cm}^{-2}\right)$ & $2.7 \pm 0.3$ & $2.7 \pm 0.2$ & $2.7 \pm 0.6$ & $3.2 \pm 0.4$ & $3.0 \pm 0.1$ \\
\hline \multirow[t]{2}{*}{ Blackbody } & $T_{\mathrm{BB}}(\mathrm{keV})$ & $1.8 \pm 0.1$ & $1.87 \pm 0.07$ & $2.1 \pm 0.2$ & $1.72 \pm 0.09$ & $1.8 \pm 0.2$ \\
\hline & $N_{\mathrm{BB}}^{\dagger \dagger}$ & $3.3 \pm 0.4$ & $3.4 \pm 0.3$ & $2.8 \pm 0.4$ & $3.5 \pm 0.4$ & $3.0 \pm 0.7$ \\
\hline \multirow{3}{*}{ Power law } & $\Gamma_{\text {pow }}^{\mathrm{BB}}$ & $3.16 \pm 0.07$ & $3.07 \pm 0.05$ & $2.98 \pm 0.08$ & $3.02 \pm 0.07$ & $2.9 \pm 0.1$ \\
\hline & $N_{\text {pow }}^{\dagger \dagger}$ & $0.03 \pm 0.06$ & $0.024 \pm 0.04$ & $0.019 \pm 0.001$ & $0.04 \pm 0.01$ & $0.02 \pm 0.01$ \\
\hline & $\chi^{2}$ (d.o.f.) & $1.12(212)$ & $1.11(333)$ & $1.01(181)$ & $0.96(127)$ & $0.62(159)$ \\
\hline phabs & $N_{\mathrm{H}}\left(\times 10^{21} \mathrm{~cm}^{-2}\right)$ & $3.5 \pm 0.6$ & $3.1 \pm 0.6$ & $2.9 \pm 0.1$ & $2.8 \pm 0.3$ & $3.0 \pm 0.1$ \\
\hline \multirow{4}{*}{ bmc } & $\Gamma_{\mathrm{bmc}}$ & $1.8 \pm 0.1$ & $1.86 \pm 0.04$ & $1.74 \pm 0.07$ & $1.81 \pm 0.06$ & $1.64 \pm 0.09$ \\
\hline & $T_{\mathrm{s}}(\mathrm{eV})$ & $140 \pm 30$ & $160 \pm 20$ & $200 \pm 40$ & $220 \pm 30$ & $210 \pm 20$ \\
\hline & $\log A$ & $0.33 \pm 0.07$ & $0.36 \pm 0.02$ & $0.34 \pm 0.05$ & $0.32 \pm 0.02$ & $0.36 \pm 0.05$ \\
\hline & $N_{\mathrm{bmc}}^{\dagger \dagger}$ & $7.56 \pm 0.04$ & $7.2 \pm 0.1$ & $6.1 \pm 0.1$ & $6.9 \pm 0.1$ & $6.58 \pm 0.05$ \\
\hline phabs*bmc & $\chi^{2}$ (d.o.f.) & $1.08(212)$ & $1.19(333)$ & $1.09(181)$ & $1.12(227)$ & 0.89 (159) \\
\hline \multirow[t]{3}{*}{ Gaussian } & $E_{\text {line }}(\mathrm{keV})$ & $6.4 \pm 0.1$ & $6.3 \pm 0.1$ & $6.4 \pm 0.2$ & $6.41 \pm 0.03$ & $6.38 \pm 0.09$ \\
\hline & $\sigma_{\text {line }}(\mathrm{eV})$ & $200 \pm 10$ & $150 \pm 60$ & $170 \pm 90$ & $110 \pm 30$ & $160 \pm 30$ \\
\hline & $N_{\text {line }}$ & $8.9 \pm 0.3$ & $3.7 \pm 0.5$ & $5.3 \pm 0.1$ & $1.6 \pm 0.2$ & $9.4 \pm 0.5$ \\
\hline phabs*(bmc+Gaussian) & $\chi^{2}$ (d.o.f.) & $1.01(209)$ & $1.12(330)$ & $1.08(178)$ & $1.07(224)$ & $0.95(156)$ \\
\hline
\end{tabular}

Notes. Errors are given at the $90 \%$ confidence level. ${ }^{(\dagger)}$ Normalization parameters of blackbody and bmc components are in units of $L_{34}^{\text {soft }} / d_{10}^{2} \mathrm{erg} \mathrm{s}^{-1} \mathrm{kpc}^{-2}$, where $L_{34}^{\text {soft }}$ is soft photon luminosity in units of $10^{34} \mathrm{erg} \mathrm{s}^{-1}, d_{10}$ is the distance to the source in units of $10 \mathrm{kpc}$, while power-law and Gaussian components are in units of $\mathrm{keV}^{-1} \mathrm{~cm}^{-2} \mathrm{~s}^{-1}$ at $1 \mathrm{keV}$ and $10^{-5} \times$ total photons $\mathrm{cm}^{-2} \mathrm{~s}^{-1}$ at $1 \mathrm{keV}$, respectively. $T_{\mathrm{BB}}$ and $T_{\mathrm{s}}$ are the temperatures of the blackbody and seed photon components, respectively (in keV and eV). $\Gamma_{\text {pow }}$ and $\Gamma_{\mathrm{bmc}}$ are the indices of the power law and bmc, respectively.

Table 3. RXTE observations of NGC 7469.

\begin{tabular}{lccc}
\hline \hline Number of set & Dates, MJD & RXTE Proposal ID & Dates UT \\
\hline R1 & $50185-50251$ & $10293,10315^{1}$ & Apr. 12-June 27, 1996 \\
R2 & $52737-53001$ & $80152^{1}$ & Apr. 8-Dec. 28, 2003 \\
R3 & $53373-54097$ & $90154,91138,92108^{1}$ & Apr. 10, 2005-Dec. 29, 2006 \\
R4 & $54836-55194$ & $94144^{1}$ & Jan. 5, 2009-Dec. 29, 2009 \\
\hline
\end{tabular}

References. (1) Rivers et al. (2013).

\section{Results}

\subsection{Images and light curves}

To avoid a possible contamination from nearby sources we used the Swift data (Gehrels et al. 2004) and made a visual inspection of the obtained image (after Gaussian filter smoothing). Swift data processing is described in our previous paper (e.g., Titarchuk \& Seifina, 2015). The Swift/XRT (0.3-10 keV) image of NGC 7469's field of view is presented in Fig. 2. The image segment selected by the yellow dashed-line box is also shown in Fig. 3 with more details. Here, contours correspond to nine logarithmic intervals in the range of $0.003-5 \%$ with respect to the brightest pixel. The image obtained during observations of NGC 7469 between April 27, 2004 and October 7, 2008 (with exposure time of $66 \mathrm{ks}$ ) is presented in Fig. 3. We point out this region as selected by the yellow dashed-line box in the larger $\left(2^{\prime} \times 2^{\prime}\right)$ Swift image in Fig. 2. Contour levels demonstrate the absence of the X-ray jet-like (elongated) structure and the minimal contamination by other point sources and diffuse emission around NGC 7469. This image (Fig. 3) shows that the X-ray emission region is extended up to $5 \mathrm{kpc}$.

Before proceeding with details of the spectral fitting, we study the long-term behavior of NGC 7469, in particular its activity patterns. We compare the one-day average X-ray and 


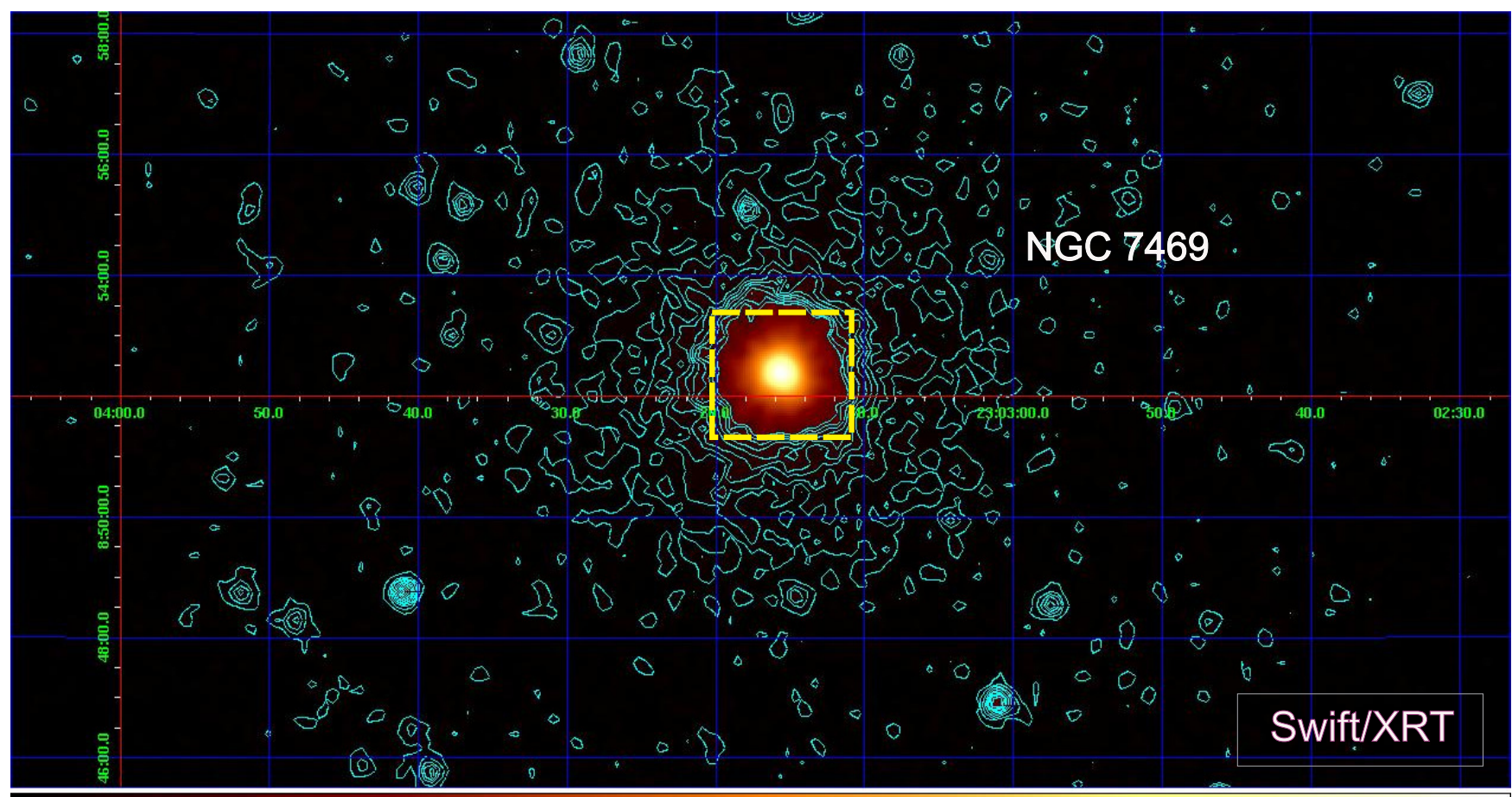

100

200

$300 \quad 400 \quad 500 \quad 60070$

Fig. 2. Swift/XRT (0.3-10 keV) image of NGC 7469 field of view. Contour levels demonstrate the absence of the X-ray jet-like (elongated) structure and the minimal contamination by other point sources and diffuse emission around NGC 7469. The image segment selected by the yellow dashedline box is also shown in Fig. 3 with more details.

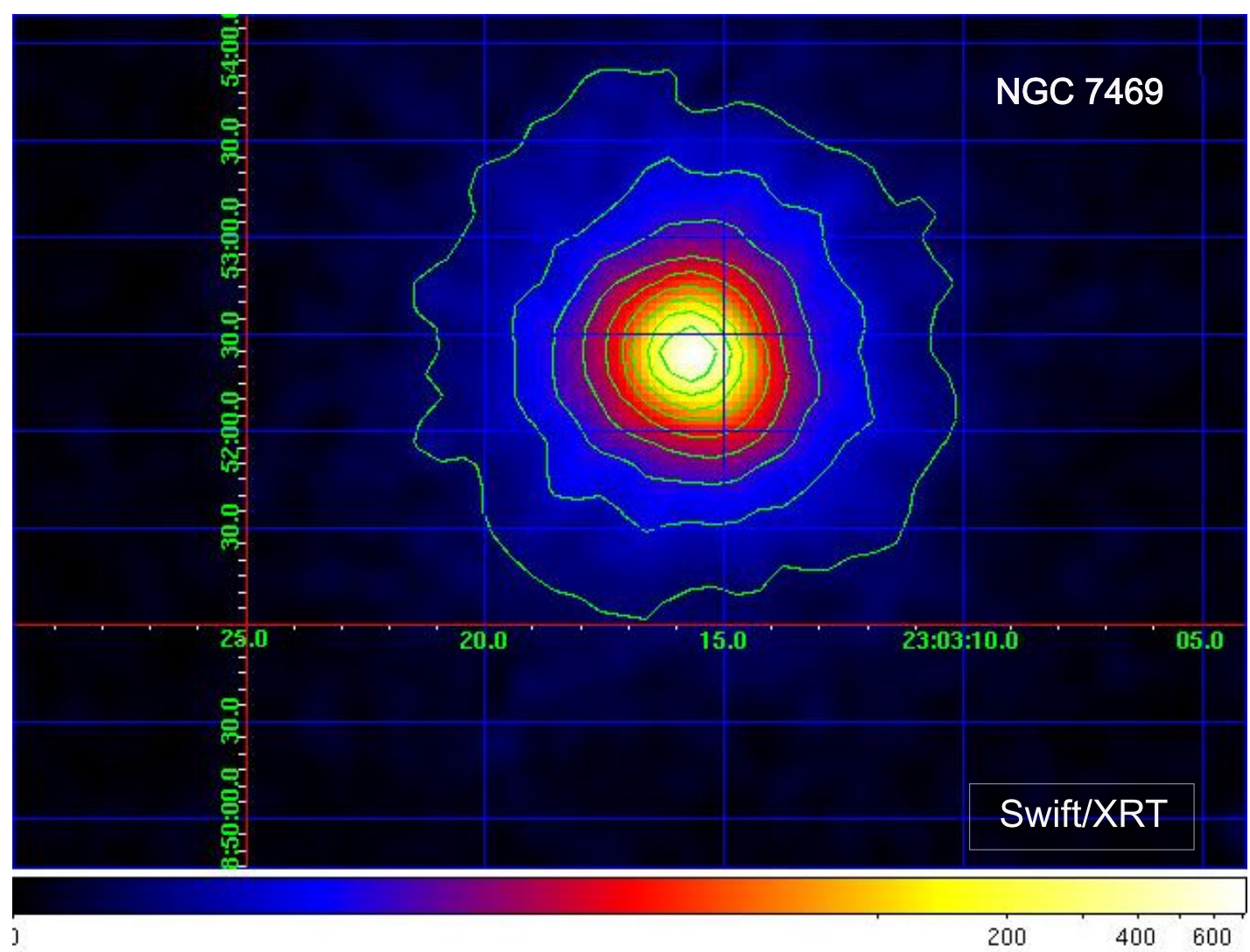

Fig. 3. Adaptively smoothed Swift/XRT image of the soft X-ray emission $(0.3-10 \mathrm{keV})$ of NGC 7469 field, of which localization is indicated by the selected yellow dashed-line box in Fig. 2. Contours correspond to nine logarithmic intervals in the range of $0.003-5 \%$ with respect to the brightest pixel, and contour levels demonstrate the minimal contamination by other point sources and diffuse emission. 
optical light curves received in the period from 1996 to 2012. We present a long-term daily light curve of NGC 7469 detected by the Assistant Store Manager (ASM) onboard the RXTE over the total life time of the mission (see top panel in Fig. 1). The bright blue triangle (arrow) indicates the ASCA observation MJD. Red points show the source signal and the blue line indicates the mean count rate level. The ASM monitoring observations are distributed more densely over time than that for an optical band. However, optical/UBV light curves of NGC 7469 show a slow (long-term) variability ( $\sim 6$ approximately six-year. Below, in the three bottom panels of Fig. 1, the optical light curves (in erg cm ${ }^{-2} \mathrm{~s}^{-1} \AA^{-1}$ ) in the $U$ (blue), $V$ (green), $B$ (red) filters are related to the 1995-2012 observations. We found at least one strong global outburst of NGC 7469 (1996-1997) peaked at around MJD $=55900$, and intervals of moderate variability with local bursts (2003-2005) and a tendency for an outburst rise towards 2012. This relatively slow variability is clearly seen in an optical/UBV range and is less evident in soft X-rays (2-12 keV, ASM).

As seen from Fig. 1, the slow variability of NGC 7469 emission is superposed with small flares at timescales larger than half a day (so called mild variability). The modulation depth in the soft X-ray and optical bands is typically $35 \%$ and $5 \%$, respectively. The optical light curves (UBV) demonstrate the highest global maximum peaking in the 1996-1997 period (see three bottom panels in Fig. 1), while the X-ray light curve shows more significant variability with frequent outbursts (top panel in Fig. 1).

It is worth noting that the peaks of X-ray outbursts in the ASM (2-12 keV) light curve are characterized by variable amplitudes, but at the maxima of the X-ray peaks they are closely related to the optical maximum (1996-1997). This fact can indicate activity of NGC 7469 in a wide photon energy range, in spite of the fact that the optical and X-rays presumably originated in geometrically different areas. The slow variability by a factor of ten has been seen in the 1996-2012 observations by ASM/RXTE. The same kind of changes of the flux were also observed in the earlier observations by Ugolkova \& Artamonov (2011). One can relate slow and mild variabilities of NGC 7469 to slow and mild changes of the mass accretion rate. Therefore, it is interesting how the slow and mild variabilities affect the spectral properties of NGC 7469.

\subsection{Spectral analysis}

Different spectral models were used in order to test them for all available data for NGC 7469. We want to establish the low/hard state (LHS) and the intermediate state evolutions using these spectral models. We investigate the ASCA and RXTE spectra to test the following XSPEC spectral models: power law, blackbody, Bulk Motion Comptonization (BMC), and their possible combinations modified by absorption and Gaussian models.

\subsubsection{Choice of the spectral model}

Our result is in agreement with previous results from ASCA. In particular, when the source was in the intermediate state, Guinazzi et al. (1994) found a photon index of $\Gamma=2.003 \pm 0.008$ (for A1 observation), while our result differs from previous results using the EXOSAT and GINGA data. The EXOSAT data fits found $\Gamma=1.78 \pm 0.07$ (see Turner \& Pounds 1987), which is close to that for the GINGA ones, $\Gamma=1.83 \pm 0.01$ (Piro et al. 1990), when the source presumably was in the low-hard state. However, these results for the ASCA, EXOSAT and GINGA data suggest a spectral variability, which is better seen in broader bandpass than those in the ASCA, EXOSAT and GINGA energy ranges.

We find that the thermal model (blackbody) fits well the low energy part, while it provides an excess emission for $E>3 \mathrm{keV}$ (e.g., for $A 5$ spectrum, $\chi_{\text {red }}^{2}=15.76$ (161 d.o.f.), see the top of Table 2). As we demonstrate, the blackbody model points towards very large absorption (greater than $3 \times 10^{21} \mathrm{~cm}^{-2}$ ), particularly for the $A 4$ spectrum, and moreover this model gives unacceptable fit quality, $\chi^{2}$ for all spectra of ASCA data. However, we must admit that the phabs*power-law model provides better fits than the thermal one. All ASCA data cannot be fitted by any single-component model. Indeed, a simple power law produces a soft excess below $0.8 \mathrm{keV}$. These significant positive residuals at low energies, less than $1 \mathrm{keV}$, suggest the presence of additional emission components in the spectrum. Thus we also test a sum of the blackbody and power-law component models. The model parameters of this combined model are $N_{\mathrm{H}}=3 \times 10^{21} \mathrm{~cm}^{-2}, k T_{\mathrm{bb}}=1.8-2.1 \mathrm{keV}$, and $\Gamma=$ 2.9 - 3.2 (see more in Table 2). The best fits of the ASCA spectra has been found using the Bulk Motion Comptonization model (BMC XSPEC model, Titarchuk et al. 1997) for which $\Gamma$ ranges from 1.6 to 1.9 for all observations (see lower part in Table 2).

We also find that a fluorescent $\mathrm{K}_{\alpha}$ line of neutral iron is clearly detected in the spectrum of NGC7469 (see Fig. 4). Adding a Gaussian profile to the absorbed BMC model improves the spectral fits. The line centroid energy and equivalent width are $E=6.4 \pm 0.01 \mathrm{keV}$ and $E W=200 \pm 70 \mathrm{eV}$, which is in agreement with Guainazzi's conclusion who apply the absorbed power-law model for ASCA spectrum, A1. However, iron-edge absorption is not detected in the NGC 7469 spectrum; the $\mathrm{K}_{\alpha}$ iron emission could be reprocessed by cold matter that surrounds the central X-ray source (Nandra \& George 1991), located far away from the central black hole. We should emphasize that all these best-fit results are found using the same model for all spectral states.

Previously, a number of papers (see, e.g., Koss 2015 and Paggi et al. 2012) considered a possible contribution from the narrow line region (NLR) in reference to Seyfert 2 galaxies. These sources are characterized by strong radio jets, while our source (NGC 7469) has a low level of radio emission without any strong indication of jet-ejection eVents. Possibly, this fact points to the low/moderate mass accretion rate in NGC 7469, and thus the matter outflow (or wind) region around the nucleus is not particularly dense as in the case of Mrk 573 (Paggi et al. 2012). Following Paggi et al. (2012) we tested an image of this galaxy in the soft X-ray emission (see Sect. 1 as well as Figs. 2 and 3) with Swift/RXT (0.3-10 keV). Contour levels demonstrate an absence of the X-ray jet-like (elongated) structure and show that $\mathrm{X}$-ray emission is extended up to $5 \mathrm{kpc}$ (Fig. 3).

Because NLR emission drops rapidly towards energies above $2 \mathrm{keV}$, our RXTE spectra (in the $3 \mathrm{keV}<E<50 \mathrm{keV}$ range) are not affected by it. However, we can test the NLR effect using the ASCA data. The estimates of the NLR contribution in Seyfert 1 galaxy NGC 7469 based on previous Chandra data (Scott et al. 2005) show that the contribution of the NLR region is low. Specifically, we assumed that this component is not variable on these timescales. While the Chandra observations were carried out in 2002, we apply their main results for spectral analysis of the ASCA data (1993, see Table 1). We included the emission lines and edge features into our spectral model and fitted the observed ASCA spectra (see Fig. 4). Here, the best-fit ASCA spectrum of NGC 7469 for the IS using the phabs*(bmc $+\mathrm{N}^{*}$ 


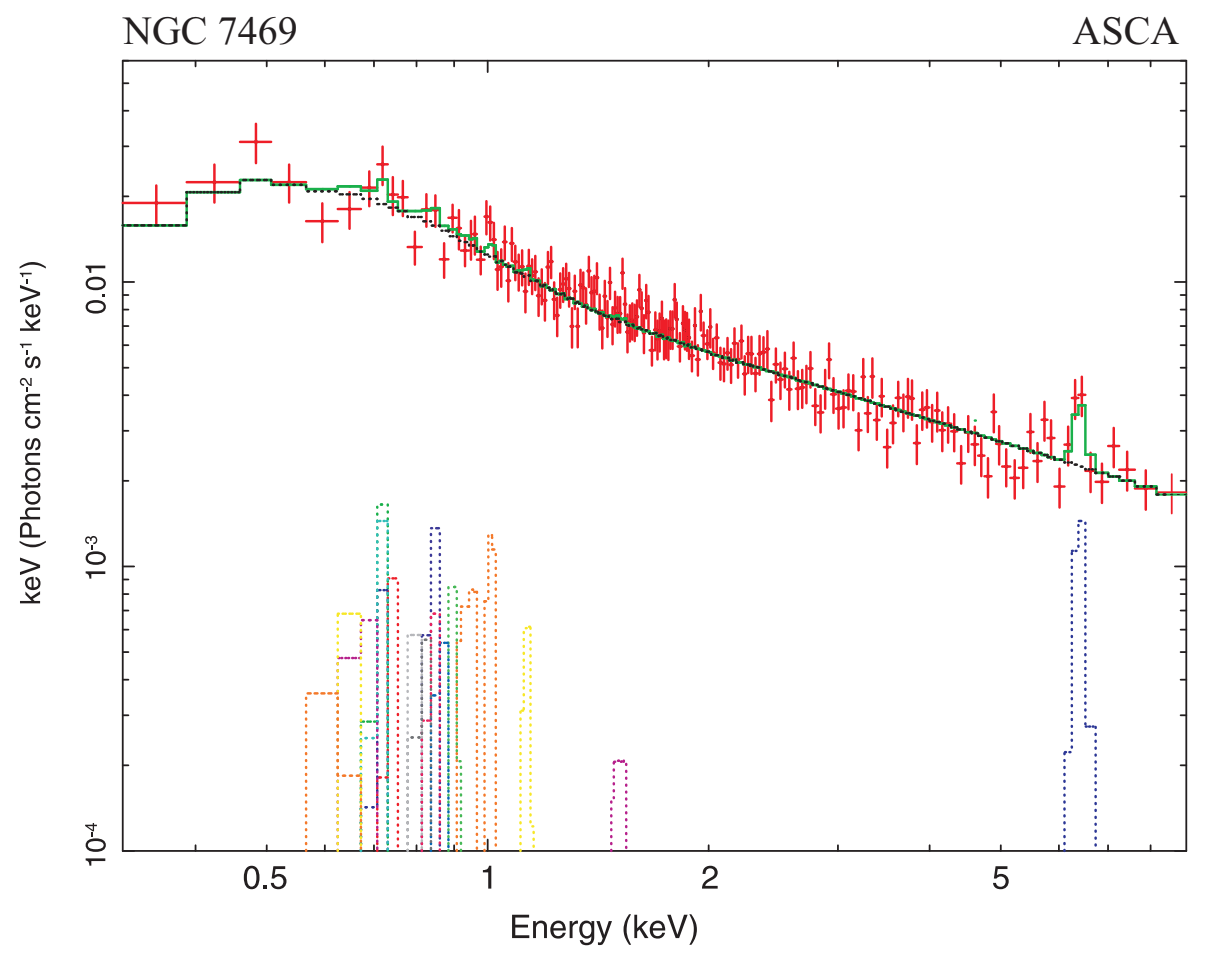

Fig. 4. Best-fit ASCA spectrum of NGC 7469 for the IS (ID=71028000) using phabs*(bmc $+\mathrm{N}^{*}$ Gaussian)*edge model $\left(\chi_{\text {red }}^{2}=1.09(137\right.$ d.o.f.)). The best-fit parameters are $\Gamma=1.8 \pm 0.1, T_{\mathrm{s}}=140 \pm 30 \mathrm{eV}$, and $E_{\text {line } 1}=6.4 \pm 0.1 \mathrm{keV}$ (see more details in Tables 2 and 4). The data are denoted by red crosses, while the spectral model presented by components is shown using green and blue lines for BMC, and Fe $\mathrm{K}_{\alpha} \mathrm{Gaussian}$ components, respectively. The narrow line components below $5 \mathrm{keV}$ are presented by different color lines.

Gaussian)*edge model $\left(\chi_{\text {red }}^{2}=1.09\right.$ for 137 d.o.f.) is presented. The best-fit parameters are $\Gamma=1.8 \pm 0.1, T_{\mathrm{s}}=140 \pm 30 \mathrm{eV}$, and $E_{\text {line1 }}=6.4 \pm 0.1 \mathrm{keV}$ (see more detalis in Tables 2 and 4 ). The data are denoted by red crosses, while the spectral model presented by components is shown using green and blue lines for BMC and $\mathrm{Fe} \mathrm{K}_{\alpha}$ Gaussian components, respectively. The narrow line components below $2 \mathrm{keV}$ are presented by different color lines. As a result, we obtained almost the same BMCnormalization (see details in Table 4) as that in the model without taking account of additional emission lines (compare the results of Tables 2 and 4). Therefore, we conclude that the narrow lines' contribution does not affect the BMC-derived $\mathrm{BH}$ mass using our scaling procedure.

It is worthwhile to note that a number of papers (e.g., Koss 2015; Paggi et al. 2012) considered the NLR effect in detail in relation to Seyfert 2 galaxies, which have strong radio jets. However, our source (NGC 7469) has very low radio emission. We also inspected the soft X-ray image of NGC 7469 Field of view (FOV) of a notable jet-like structure around NGC 7469.

Figure 5 shows the best-fit model of the spectrum of NGC 7469 (top panel). Data are taken from ASCA observations (ID $=15030000)$ carried out on June 26-27, 1994 when the source was in the low/hard state. The data are shown by black crosses and the spectral model is displayed by red line In the bottom panel we show the corresponding $\Delta \chi$ versus photon energy (in keV). Using the ASCA data we find that the seed temperatures $k T_{\mathrm{s}}$ of the $B M C$ model vary only slightly from 140 to $200 \mathrm{eV}$.

We also use the phabs*(bmc+Gaussian) model to fit all RXTE data. In order to fit all of these spectra, we use neutral column $N_{\mathrm{H}}$, which is fixed at $3 \times 10^{21} \mathrm{~cm}^{-2}$ (see also Behar et al. 2017; Wakker et al. 2011; Scott et al. 2005. In Fig. 6 we demonstrate three representative $E F_{\mathrm{E}}$ spectral diagrams for different states of NGC 7469. Data are taken from RXTE observations 80152-05-04-00 (green line), 10293-01-01-09 (red line), and 10315-01-15-10 (blue line).

In Fig. 7 we show a suggested geometry for NGC 7469 derived using our X-ray spectral analysis, where one can see that the soft (disk) photons illuminate the Compton cloud (CC) surrounding a $\mathrm{BH}$ and finally accreting matter from the $\mathrm{CC}$ leads to a $\mathrm{BH}$ through the Bulk Comptonization region (converging flow).

In Fig. 8 we present the evolution of X-ray/optical properties of NGC 7469: the RXTE/ASM count rate (top panel), the optical V-flux (in stellar magnitudes), the Comptonized fraction $f$, and the BMC normalization during the 2003 outburst transition set (R2). In the last bottom panel, we present an evolution of the photon index $\Gamma=\alpha+1$. It can be seen that the soft $\mathrm{X}$-ray flash (MJD 52800) is accompanied by a normalization $N_{\text {BMC }}$ rise at the constant value of the photon index, $\Gamma \sim 2$. The high X-ray flux (MJD 52750-52800) is seen when the Comptonized fraction $f$ is low and $\Gamma \sim 2$, while the subsequent transition of NGC 7469 from the high X-ray flux phase to the low $\mathrm{X}$-ray flux phase (MJD > 52800) is associated with an increase of the Comptonized fraction $f$ and with a decrease of the photon index $(\Gamma \sim 1.2)$ and $N_{\mathrm{BMC}}$. It is interesting that the optical variability (e.g., MJD 52850-53000) is weakly related with Xray variability. This could indicate different origins of the optical and X-ray emissions.

Applying the ASCA observations (see blue squares in Fig. 9), we find that the spectral index $\alpha$ monotonically increases from 0.6 to 0.8 (or $\Gamma$ from 1.6 to 1.8 ), when the normalization of the BMC We illustrate this index versus mass accretion rate behavior in Fig. 9 using both RXTE and ASCA observations (red triangles and blue squares, respectively). 
Table 4. Best-fit parameters of the ASCA spectra of NGC 7469 in the $0.45-10 \mathrm{keV}$ range using the model: phabs*(bmc+N*Gaussian)*edge.

\begin{tabular}{|c|c|c|c|c|c|c|c|}
\hline Model & Parameter & & 71028000 & 71028010 & 71028030 & 15030000 & 18931126 \\
\hline phabs & $N_{\mathrm{H}}\left(\times 10^{21} \mathrm{~cm}^{-2}\right)$ & & $3.5 \pm 0.6$ & $3.0 \pm 0.9$ & $2.7 \pm 0.1$ & $3.0 \pm 0.2$ & $3.1 \pm 0.1$ \\
\hline \multirow[t]{4}{*}{ bmc } & $\Gamma_{\mathrm{bmc}}$ & & $1.8 \pm 0.1$ & $1.86 \pm 0.04$ & $1.74 \pm 0.07$ & $1.81 \pm 0.06$ & $1.64 \pm 0.09$ \\
\hline & $T_{\mathrm{s}}(\mathrm{eV})$ & & $140 \pm 30$ & $160 \pm 20$ & $200 \pm 40$ & $220 \pm 30$ & $210 \pm 20$ \\
\hline & $\log A$ & & $0.33 \pm 0.05$ & $0.35 \pm 0.04$ & $0.3 \pm 0.1$ & $0.35 \pm 0.07$ & $0.4 \pm 0.1$ \\
\hline & $N_{\mathrm{hm}}^{\dagger \dagger}$ & & $7.5 \pm 0.1$ & $7.1 \pm 0.2$ & $6.0 \pm 0.1$ & $6.7 \pm 0.2$ & $6.4 \pm 0.1$ \\
\hline \multirow[t]{21}{*}{ Gaussian $^{\dagger+\dagger}$} & $E_{\text {line1 }}(\mathrm{keV})$ & $\mathrm{Fe} \mathrm{K}_{\alpha}$ & $6.4 \pm 0.1$ & $6.31 \pm 0.09$ & $6.4 \pm 0.1$ & $6.42 \pm 0.04$ & $6.38 \pm 0.09$ \\
\hline & $\sigma_{\text {line1 }}(\mathrm{eV})$ & & $200 \pm 10$ & $150 \pm 60$ & $170 \pm 90$ & $110 \pm 30$ & $160 \pm 30$ \\
\hline & $N_{\text {line } 1}$ & & $8.9 \pm 0.3$ & $3.7 \pm 0.5$ & $5.3 \pm 0.1$ & $1.6 \pm 0.2$ & $9.4 \pm 0.5$ \\
\hline & $E_{\text {line2 }}(\mathrm{keV})$ & & $1.5 \pm 0.1$ & $1.51 \pm 0.08$ & $1.52 \pm 0.09$ & $1.53 \pm 0.06$ & $1.50 \pm 0.09$ \\
\hline & $E_{\text {line3 }}(\mathrm{keV})$ & $\ldots$ & $1.13 \pm 0.09$ & $1.14 \pm 0.07$ & $1.14 \pm 0.02$ & $1.12 \pm 0.03$ & $1.12 \pm 0.08$ \\
\hline & $E_{\text {line4 }}(\mathrm{keV})$ & Ne X Ly $\alpha$ & $1.03 \pm 0.07$ & $1.03 \pm 0.08$ & $1.0 \pm 0.1$ & $1.04 \pm 0.05$ & $1.02 \pm 0.07$ \\
\hline & $E_{\text {line5 }}(\mathrm{keV})$ & & $0.94 \pm 0.06$ & $0.93 \pm 0.04$ & $0.94 \pm 0.09$ & $0.94 \pm 0.08$ & $0.93 \pm 0.09$ \\
\hline & $E_{\text {line6 }}(\mathrm{keV})$ & $\mathrm{Ne} I X$ & $0.91 \pm 0.08$ & $0.90 \pm 0.08$ & $0.9 \pm 0.1$ & $0.91 \pm 0.07$ & $0.91 \pm 0.08$ \\
\hline & $E_{\text {line7 }}(\mathrm{keV})$ & O XIII Ly7 & $0.86 \pm 0.09$ & $0.87 \pm 0.07$ & $0.86 \pm 0.07$ & $0.86 \pm 0.04$ & $0.86 \pm 0.09$ \\
\hline & $E_{\text {line8 }}(\mathrm{keV})$ & O XIII Ly6 & $0.85 \pm 0.07$ & $0.85 \pm 0.09$ & $0.85 \pm 0.06$ & $0.84 \pm 0.09$ & $0.85 \pm 0.07$ \\
\hline & $E_{\text {line9 }}(\mathrm{keV})$ & O XIII Ly5 & $0.84 \pm 0.08$ & $0.84 \pm 0.03$ & $0.84 \pm 0.02$ & $0.84 \pm 0.07$ & $0.83 \pm 0.08$ \\
\hline & $E_{\text {line10 }}(\mathrm{keV})$ & $\ldots$ & $0.8 \pm 0.1$ & $0.81 \pm 0.05$ & $0.80 \pm 0.06$ & $0.81 \pm 0.09$ & $0.81 \pm 0.09$ \\
\hline & $E_{\text {line11 }}(\mathrm{keV})$ & $\ldots$ & $0.79 \pm 0.05$ & $0.79 \pm 0.09$ & $0.78 \pm 0.06$ & $0.77 \pm 0.02$ & $0.78 \pm 0.08$ \\
\hline & $E_{\text {line12 }}(\mathrm{keV})$ & O VII & $0.74 \pm 0.03$ & $0.75 \pm 0.08$ & $0.74 \pm 0.05$ & $0.74 \pm 0.06$ & $0.75 \pm 0.09$ \\
\hline & $E_{\text {line13 }}(\mathrm{keV})$ & $\ldots$ & $0.73 \pm 0.02$ & $0.73 \pm 0.09$ & $0.73 \pm 0.02$ & $0.73 \pm 0.03$ & $0.73 \pm 0.08$ \\
\hline & $E_{\text {line14 }}(\mathrm{keV})$ & $\ldots$ & $0.72 \pm 0.08$ & $0.72 \pm 0.04$ & $0.72 \pm 0.08$ & $0.72 \pm 0.05$ & $0.72 \pm 0.09$ \\
\hline & $E_{\text {line15 }}(\mathrm{keV})$ & $\ldots$ & $0.71 \pm 0.04$ & $0.71 \pm 0.06$ & $0.70 \pm 0.09$ & $0.69 \pm 0.09$ & $0.71 \pm 0.07$ \\
\hline & $E_{\text {line16 }}(\mathrm{keV})$ & $\ldots$ & $0.67 \pm 0.09$ & $0.66 \pm 0.08$ & $0.67 \pm 0.02$ & $0.68 \pm 0.07$ & $0.68 \pm 0.09$ \\
\hline & $E_{\text {line17 }}(\mathrm{keV})$ & O VIII Ly $\alpha$ & $0.65 \pm 0.07$ & $0.65 \pm 0.04$ & $0.64 \pm 0.09$ & $0.64 \pm 0.06$ & $0.65 \pm 0.08$ \\
\hline & $E_{\text {line18 }}(\mathrm{keV})$ & & $0.61 \pm 0.08$ & $0.61 \pm 0.04$ & $0.6 \pm 0.1$ & $0.61 \pm 0.05$ & $0.60 \pm 0.04$ \\
\hline & $E_{\text {line19 }}(\mathrm{keV})$ & OVII & $0.62 \pm 0.05$ & $0.62 \pm 0.07$ & $0.62 \pm 0.09$ & $0.63 \pm 0.07$ & $0.62 \pm 0.08$ \\
\hline \multirow[t]{3}{*}{ edge } & $E_{\text {edge }}(\mathrm{keV})$ & Si XIV Ly $\alpha$ & $2.0 \pm 0.1$ & $2.01 \pm 0.06$ & $2.0 \pm 0.2$ & $2.04 \pm 0.06$ & $2.03 \pm 0.09$ \\
\hline & $\tau_{\max }\left(\times 10^{-3}\right)$ & & $7.43 \pm 0.06$ & $6.1 \pm 0.1$ & $6.5 \pm 0.2$ & $5.1 \pm 0.3$ & $6.0 \pm 0.1$ \\
\hline & $\chi^{2}$ (d.o.f.) & & 1.09 (137) & $1.01(268)$ & 1.07 (116) & 1.09 (162) & $0.82(94)$ \\
\hline
\end{tabular}

Notes. Errors are given at the $90 \%$ confidence level. ${ }^{(\dagger \dagger)}$ Normalization parameters of BMC component is in units of $L_{34}^{\text {soft }} / d_{10}^{2} \mathrm{erg} \mathrm{s}^{-1} \mathrm{kpc}^{-2}$, where $L_{34}^{\text {soft }}$ is soft photon luminosity in units of $10^{34} \mathrm{erg} \mathrm{s}^{-1}, d_{10}$ is the distance to the source in units of $10 \mathrm{kpc}$, while the Gaussian component is in units of $\mathrm{keV}^{-1} \mathrm{~cm}^{-2} \mathrm{~s}^{-1}$ at $1 \mathrm{keV}$ and $10^{-5} \times$ total photons $\mathrm{cm}^{-2} \mathrm{~s}^{-1}$ at $1 \mathrm{keV} . T_{\mathrm{s}}$ is the temperature of the seed photon component (in keV), while $\Gamma_{\text {bme }}$ is the index of the BMC component. ${ }^{\dagger \dagger \dagger}$ For the rest of the Gaussian-line components, $\sigma_{\text {line }}<100 \mathrm{eV}$ and $N_{\text {line }}<10^{-5}$ total photons $\mathrm{cm}^{-2} \mathrm{~s}^{-1}$ at $1 \mathrm{keV}$.

\subsubsection{Spectral modeling for NGC 7469}

As a result of the model selection (see Sect. 3.2.1), we assume a model that consists of a sum of the Comptonization (BMC) component and the emission line (Gaussian) component to fit all spectral data (Tables 1 and 3). We briefly remind the reader of the physical picture described by the BMC model (see Titarchuk et al. 1997), its basic assumptions and parameters. The BMC Comptonization spectrum is a sum of a part of the blackbody directly observed by the Earth observer (a fraction of $1 /(1+A)$ ) and a fraction of the blackbody, $f=A /(1+A)$, convolved with the upscattering Green's function $G\left(E, E_{0}\right)$, which is, in the $\mathrm{BMC}$ approximation, a broken power law

$\left.F_{v}=C_{\mathrm{N}}\left\{B B(E)+f * \int_{0}^{\infty}\left[B B\left(E_{0}\right) * G\left(E, E_{0}\right)\right] \mathrm{d} E_{0}\right]\right\}$.

It is worthwhile emphasizing that the Green's function is characterized by only one parameter, the spectral index $\alpha=\Gamma-1$. Consequently, one can see that the BMC model has the main parameters, $\alpha, A$, the seed blackbody temperature $T_{\mathrm{s}}$, and blackbody normalization, which is proportional to the seed blackbody luminosity and inversely proportional to $d^{2}$, where $\mathrm{d}$ is a distance to the source. We also apply a multiplicative phabs component characterized by an equivalent hydrogen column $N_{\mathrm{H}}$ in order to take into account absorption by neutral material. The parameters of the Gaussian line component are the energy $E_{\text {line }}$ of the line centroid, the line width $\sigma_{\text {line }}$, and the line normalization $N_{\text {line }}$.

One can clearly see spectral evolution from the LHS to the IS in Fig. 8. The BMC model successfully fits the NGC 7469 spectra for all spectral states. Particularly, the ASCA spectrum obtained during the observation with ID $=15030000$ fit using the BMC model is shown in Fig. 5. In Table 2 (at the bottom), we present the results of spectral fitting ASCA data of NGC 7469 using our main spectral model phabs*(bmc+Gaussian). Using the RXTE data, the LHS-IS transition is related to the photon index, $\Gamma$, which changes from 1.2 to 2.1 when the normalization of the seed photon increases. For the RXTE fits, we fix the seed photon temperature at $200 \mathrm{eV}$. The BMC normalization, $N_{\text {bmc }}$, varies by a factor of thirty in the range of $0.1<N_{\mathrm{BMC}}<$ $3.3 \times L_{34} / d_{10}^{2} \mathrm{erg} \mathrm{s}^{-1} \mathrm{kpc}^{-2}$, while the Comptonized (illumination) fraction changes in a wide range of $0.3<\log A<0.7$ $[f=A /(1+A)]$.

As we have already discussed above, the spectral evolution of NGC 7469 has been previously investigated using X-ray data. In particular, Guinazzi et al. (1994) and Rivers et al. (2013) studied the 1993 (ASCA) and 1996-2009 (RXTE) data sets, respectively 


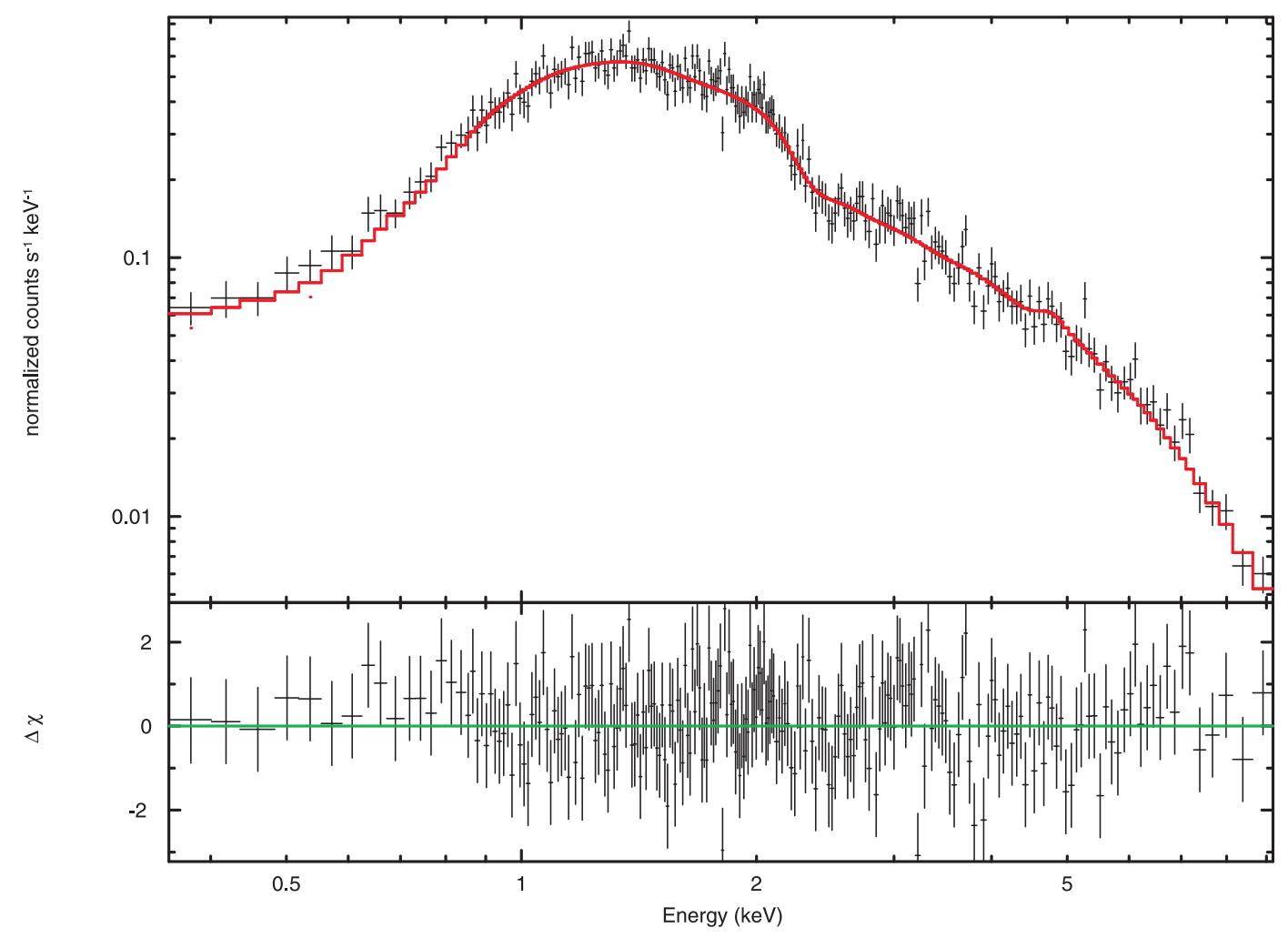

Fig. 5. Best-fit modeling of the spectrum of NGC 7469 (top panel) with $\Delta \chi$ residuals (bottom panel). Data are taken from ASCA observations $(\mathrm{ID}=15030000)$ carried out on 1994 June $26-27$, when the source was in the low/hard state.

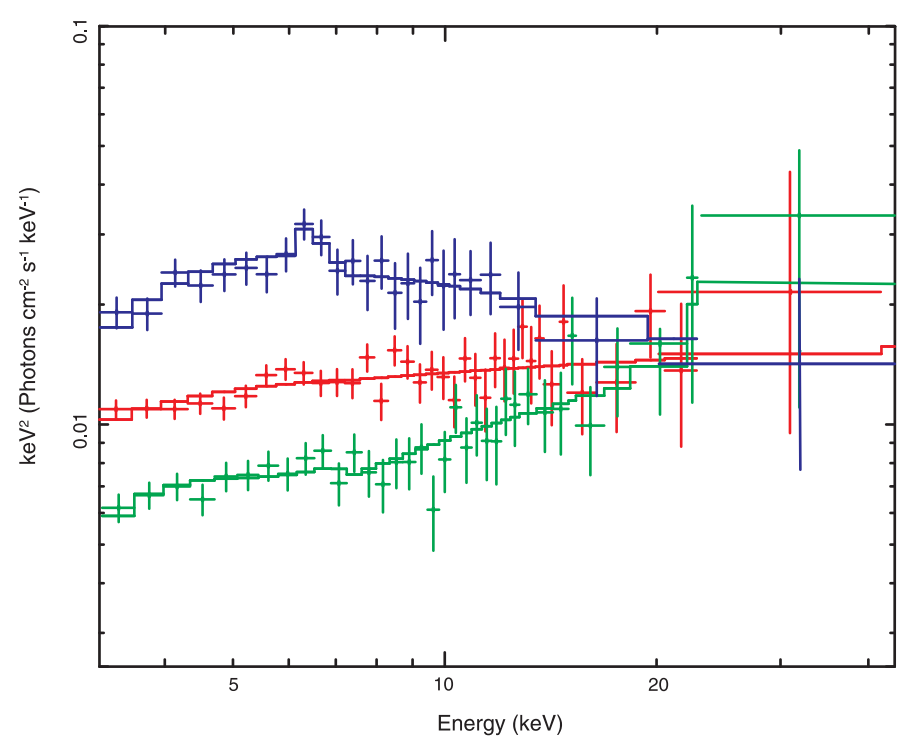

Fig. 6. Three representative $E F_{\mathrm{E}}$ diagrams for different states of NGC 7469. Data are taken from RXTE observations 80152-05-04-00 (green line), 10293-01-01-09 (red line), and 10315-01-15-10 (blue line).

(see also Tables 1 and 3) using an additive diskbb plus power-law model and a simple power-law model, respectively. These qualitative models describe an evolution of these spectral model parameters throughout state transitions during the outbursts.

We also found a similar spectral behavior using our model and the full set of the RXTE observations. In particular, as in the aforementioned papers by Guainazzi and Rivers, we have also revealed that NGC 7469 demonstrates a change of the photon index $\Gamma$ between $\sim 1.2$ and 2.2 during the LHS-IS transition. In addition, we revealed that $\Gamma$ tends to saturate at 2.1 at high values of $N_{\text {bmc }}$. In other words, we found that $\Gamma$ saturates at 2.1 when the mass accretion rate increases.

Our spectral model shows a very good performance throughout all data sets. In Table 2 and Figs. 4-5 we demonstrate a good performance of the BMC model in application to the ASCA and RXTE data for which the reduced $\chi_{\text {red }}^{2}=\chi^{2} / N_{\text {d.o.f. }}\left(N_{\text {d.o.f. }}\right.$ is the number of degree of freedom) is less or around 1 for all observations $\left(0.95<\chi_{\text {red }}^{2}<1.12\right)$.

We can also estimate a radius of the blackbody emission region. We find the blackbody radius $R_{\mathrm{BB}}$ using the relation $L_{\mathrm{BB}}=4 \pi R_{\mathrm{BB}}^{2} \sigma T_{\mathrm{BB}}^{4}$, where $L_{\mathrm{BB}}$ is the seed blackbody luminosity and $\sigma$ is the Stefan-Boltzmann constant. With a distance $D$ to the source of $70 \mathrm{Mpc}$, we find the region associated with the blackbody radius of $R_{\mathrm{BB}} \sim 4 \times 10^{11} \mathrm{~cm}$. Such an extensive blackbody region should only be around a SMBH and thus NGC 7469 is probably a SMBH source. We remind the reader that $R_{\mathrm{BB}}$ is of the order of $10^{6} \mathrm{~cm}$ for a Galactic $\mathrm{BH}$ of a mass around ten solar masses (see STS14) and correspondingly $R_{\mathrm{BB}} \sim 4 \times 10^{11}$ should correspond to a characterisic size of the order of $10^{6}$ solar masses. We also establish that the photon index $\Gamma$ correlates with the BMC normalization, $N_{\mathrm{BMC}}$ (proportional to the mass accretion rate, $\dot{M}$ ) and finally saturates at higher values of $\dot{M}$ (see Fig. 9). The index $\Gamma$ monotonically grows from 1.2 to 2.1 with the $\dot{M}$ increase and then saturates at $\Gamma_{\text {sat }}=2.0 \pm 0.1$ for high values of $\dot{M}$.

\section{Discussion}

\subsection{Saturation of the index as a signature of a $\mathrm{BH}$}

Having applied our analysis to an evolution of the photon index $\Gamma$ in NGC 7469, we find the photon index, $\Gamma$, saturates with the 


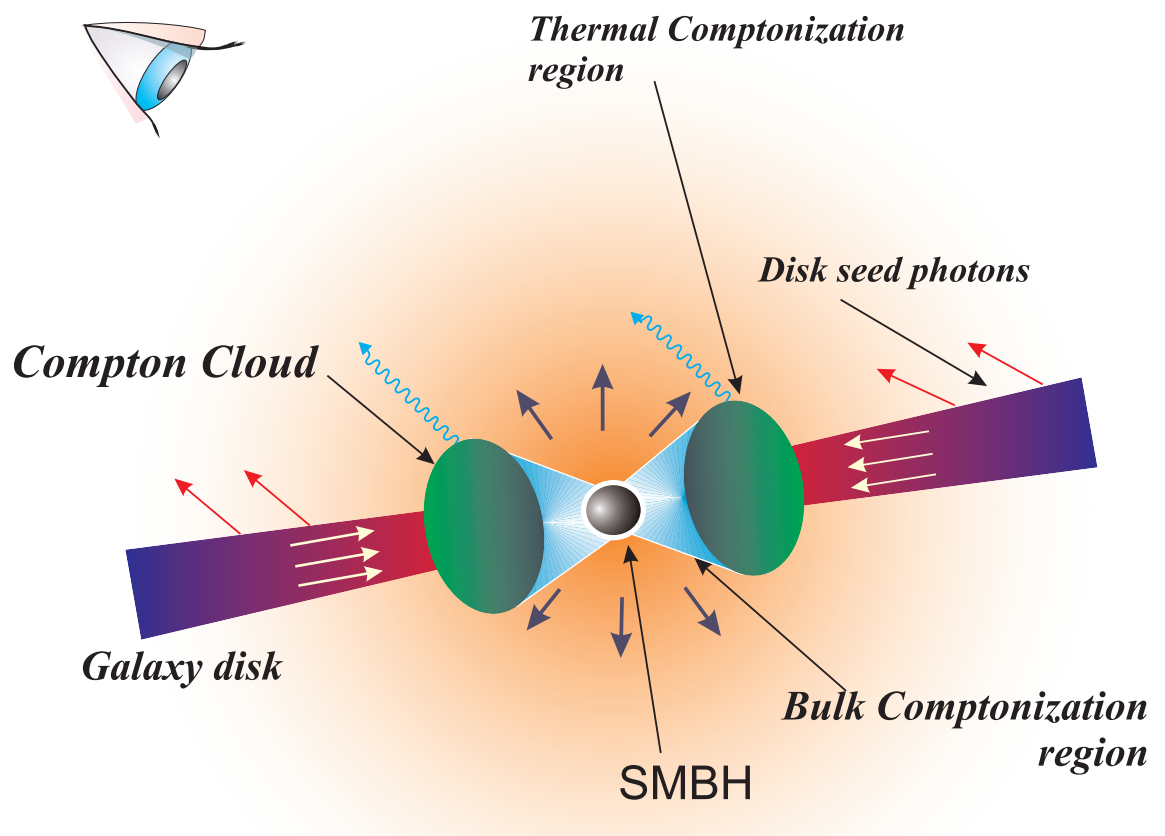

Fig. 7. Suggested geometry for NGC 7469. Disk soft photons are upscattered (Comptonized) off the relatively hot plasma of the transition layer (Compton cloud). mass accretion rate, $\dot{M}$. ST09 demonstrates that this index saturation is a first indication of the converging flow into a $\mathrm{BH}$.

In their early paper, Titarchuk et al. (1998) demonstrated using the equation of motion that the innermost part of the accretion flow (the so-called transition layer), shrinks when $\dot{M}$ increases. It is worthwhile emphasizing that for a $\mathrm{BH}, \Gamma$ increases and finally saturates for high $\dot{M}$. Titarchuk \& Zannias (1998), hereafter TZ98, semi-analytically discovered the saturation effect and later Laurent \& Titarchuk (1999, 2011), hereafter LT99 and LT11, confirmed this effect making Monte Carlo simulations.

Observations of many Galactic BHs (GBHs) and their X-ray spectral analysis (see ST09, Titarchuk \& Seifina 2009; Seifina \& Titarchuk 2010; Seifina et al. 2014; hereafter TS14) demonstrate a confrmation of this TZ98 prediction. For our particular source, NGC 7469, we also reveal that $\Gamma$ monotonically increases from 1.2 and then finally saturates at a value of 2.1 (see Fig. 9). The index $-\dot{M}$ correlation found in NGC 7469 allows us to estimate a BH mass in this source by scaling this correlation with those detected in a number of GBHs and extragalactic sources (see details below, in Sect. 4.3).

\subsection{An estimate of a BH mass in NGC 7469}

In order to estimate $\mathrm{BH}$ mass, $M_{\mathrm{BH}}$ of NGC 7469, we chose two Galactic sources (GRO J1655-40, Cygnus X-1 (see ST09)) and the extragalactic source NGC 4051 (Seifina et al. 2018; hereinafter SCT18), as the reference sources, whose BH masses and distances are now well established.

A BH mass for GRO J1655-40 is estimated by dynamical methods. For a BH mass estimate of NGC 7469, we also use the BMC normalizations, $N_{\mathrm{BMC}}$, of these reference sources. As a result, we scale the index versus $N_{\mathrm{BMC}}$ correlations for these reference sources with that of the target source NGC 7469 (see Fig. 10). The value of the index saturation is almost the same, $\Gamma \sim 2$, for all these target and reference sources. We apply the correlations found in these four reference sources to make a comprehensive cross-check of a BH mass estimate for NGC 7469.

The main points of our scaling method are as follows. Shaposhnikov \& Titarchuk (2007), hereafter ST07, used an inverse proportionality of a frequency of quasi-periodic oscilations (QPO) on BH mass in order to estimate a BH mass. ST07 also present theoretical arguments in terms of the transition layer model that predict the inverse dependence of QPO frequencies on BH mass. Therefore, as a first scaling law we write

$s_{v}=\frac{v_{\mathrm{r}}}{v_{\mathrm{t}}}=\frac{M_{\mathrm{t}}}{M_{\mathrm{r}}}$

where subscripts $r$ and $t$ denote reference and target sources in scaling, respectively.

The second scaling law, which we use as a basis for our analysis technique, is a fundamental principle of inverse square intensity dependence on the source distance, which is expressed by the equation

$\frac{F_{\mathrm{r}}}{F_{\mathrm{t}}}=\frac{L_{\mathrm{r}}}{L_{\mathrm{t}}} \frac{d_{t}^{2}}{d_{r}^{2}}$.

Here $F$ stands for the source flux detected by an observer on Earth, $L$ is source luminosity, and $d$ is source distance. The luminosity $L$ can be represented as

$L=\frac{G M_{\mathrm{BH}}}{R_{*}} \dot{M} \eta \sim \frac{G M_{\mathrm{BH}}}{R_{S}} \dot{M} \eta \sim \dot{M} \eta=M_{\mathrm{BH}} \dot{m} \eta$,

where $R_{*}$ is an effective radius at which energy release occurs, $\eta$ is the efficiency of gravitation energy conversion into radiation power, $\dot{M}$ is the accretion rate, and $\dot{m}$ is its dimensionless analog normalized by the Eddington luminosity. Both $\dot{m}$ and $\eta$ are considered to be the same for two different sources in the same spectral state, which leads to $L_{\mathrm{r}} / L_{\mathrm{t}}=M_{\mathrm{r}} / M_{\mathrm{t}}=1 / s_{v}$. In our analysis of energy spectra from $\mathrm{BH}$ sources, we determine the normalization of seed radiation, which is supplied by an accretion flow (disk) prior to Comptonization. The ratio of this normalization for two sources in the same spectral state can be written as

$s_{\mathrm{N}}=\frac{N_{\mathrm{r}}}{N_{\mathrm{t}}}=\frac{L_{\mathrm{r}}}{L_{\mathrm{t}}} \frac{d_{\mathrm{t}}^{2}}{d_{\mathrm{r}}^{2}} f_{\mathrm{G}}$

Here $f_{\mathrm{G}}$ is a geometry factor that comes due to the fact that the accretion disk, which produces thermal input for Comptonization, has a plane geometry. Therefore, in the case of radiation coming directly from the disk, it would have the value 


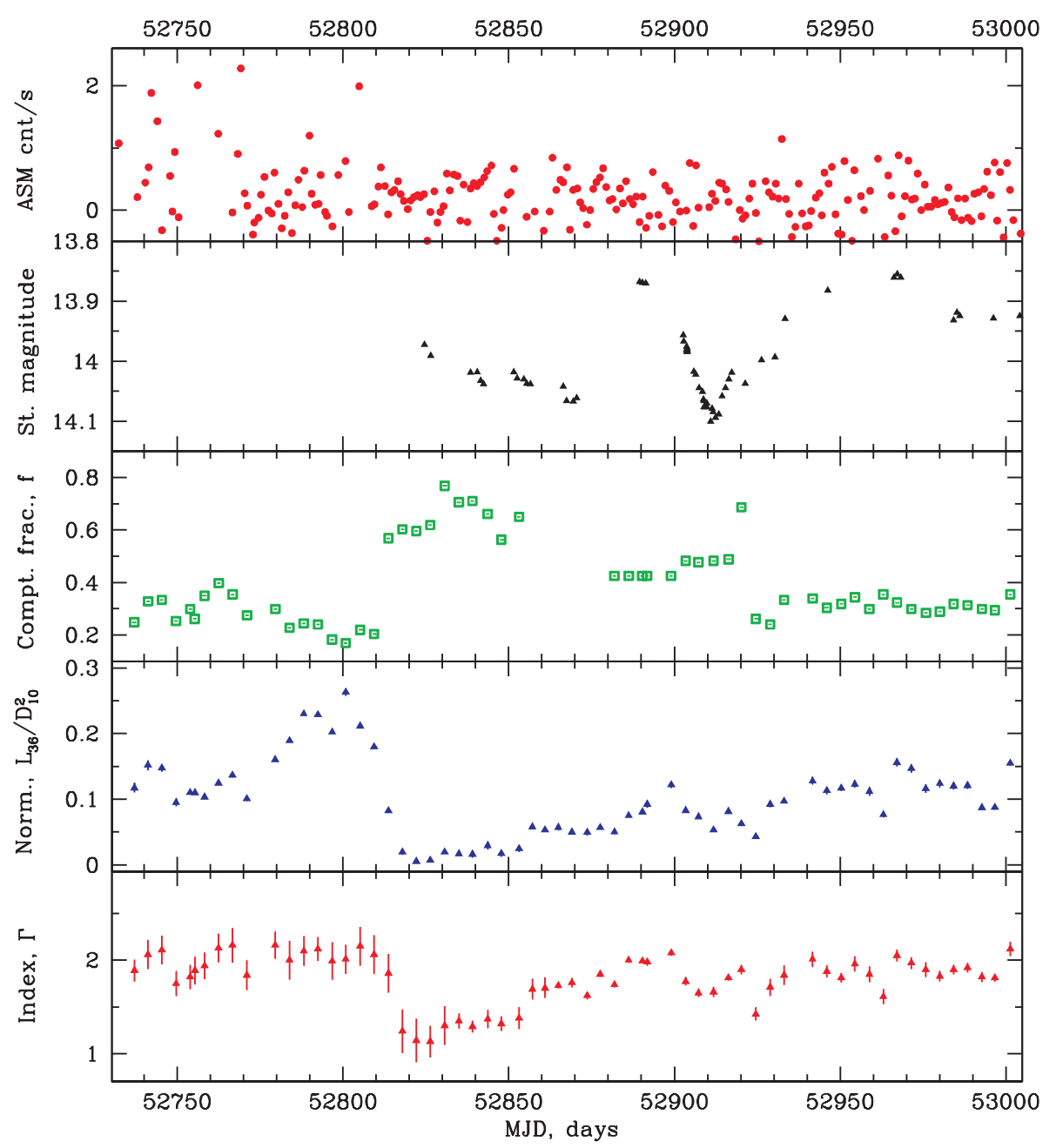

Fig. 8. From top to bottom: evolutions of the RXTE/ASM count rate, the optical V-flux (in stellar magnitudes), the Comptonized fraction $f$, and the BMC normalization during the 2003 outburst transition set $(R 2)$. In the last bottom panel, we present an evolution of the photon index $\Gamma=\alpha+1$.

$f_{\mathrm{G}}=\left(\cos i_{\mathrm{d}}\right)_{\mathrm{r}} /\left(\cos i_{\mathrm{d}}\right)_{\mathrm{t}}$, where $\cos i_{\mathrm{d}}$ is the inclination angle of the disk. When the information on the system inclination is available, we can use these $i_{\mathrm{d}}$ values.

We are now in a position to write down the final equations of our scaling analysis. Namely, when $s_{v}$ and $s_{\mathrm{N}}$ are measured, the mass and the distance of the target source can be calculated as

$M_{\mathrm{t}}=s_{v} M_{\mathrm{r}}$

and

$d_{\mathrm{t}}=d_{\mathrm{r}}\left(\frac{s_{v} s_{\mathrm{N}}}{f_{\mathrm{G}}}\right)^{1 / 2}$.

This equation allows us to estimate $s_{v}=M_{\mathrm{t}} / M_{\mathrm{t}}$ using values of $s_{\mathrm{N}}, f_{\mathrm{G}}, d_{\mathrm{t}}$, and $d_{\mathrm{r}}$.

With Eqs. (6) and (7) in hand, the task of BH-mass and distance measurements for a target source is reduced to the determination of scaling coefficients $s_{v}$ and $s_{\mathrm{N}}$ with respect to the data for a reference source. This is achieved by a technique similar to that adopted by ST07. Specifically, after scalable state transition episodes are identified for two sources, the correlation pattern for a reference transition is parameterized in terms of the analytical function (see also ST09)

$F(x)=\mathcal{A}-(\mathcal{D} \cdot \mathcal{B}) \ln \left\{\exp \left[\left(1.0-\left(x / x_{\text {tr }}\right)^{\beta}\right) / \mathcal{D}\right]+1\right\}$,

with $x=N_{\text {bmc }}$.
As can be seen from Fig. 10, the correlations of the target source (NGC 7469) and the reference sources are characterized by similar shapes and index saturation levels. Consequently, it allows us to make a reliable scaling of these correlations with that of NGC 7469. In order to implement the scaling technique, we introduce an analytical approximation of the $\Gamma-N_{\text {bmc }}$ correlation, fit by a formula (8).

As a result of fitting the observed correlation by this function $F(x)$, we obtained a set of the best-fit parameters $\mathcal{A}, \mathcal{B}$, $\mathcal{D}, N_{\text {tr }}$, and $\beta$ (see Table 5). The meaning of these parameters is described in detail in our previous paper (Titarchuk \& Seifina 2016; hereafter TS16). This function $F(x)$ is widely used for a description of the correlation of $\Gamma$ versus $N_{\mathrm{bmc}}$ (Sobolewska \& Papadakis 2009; ST09, Seifina \& Titarchuk 2010; STS14, Giacche et al. 2014, Titarchuk \& Seifina 2016, 2017; Seifina et al. 2017, 2018).

In order to perform this $\mathrm{BH}$ mass determination for the target source, one should rely upon the same shape of the $\Gamma-N_{\mathrm{bmc}}$ correlations for this target source and those for the reference sources. Accordingly, the only difference in values of $N_{\mathrm{bmc}}$ for these three sources is in the ratio of $\mathrm{BH}$ mass to the squared distance, $M_{\mathrm{BH}} / d^{2}$. As one can see from Fig. 10, the index saturation value $\mathcal{A}$ is approximately the same for the target and reference sources (see also the second column in Table 5). eVidently, for instance, the shape of the correlations for NGC 7469 (green line) and Cyg X-1 (black line) are similar and the only difference 


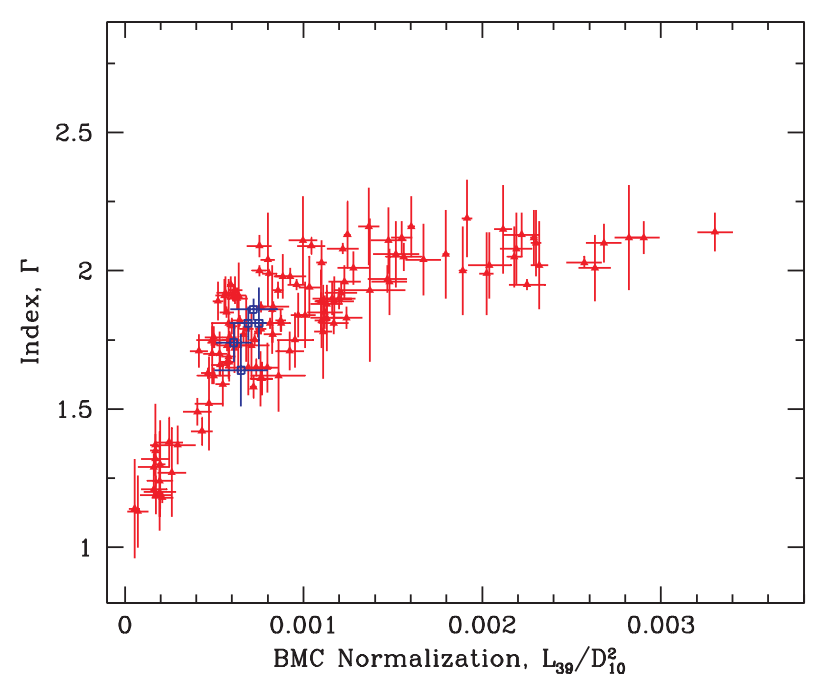

Fig. 9. Correlations of the photon index $\Gamma(=\alpha+1)$ versus the BMC normalization, $N_{\mathrm{BMC}}$ (proportional to mass accretion rate) in units of $L_{39} / D_{10}^{2}$. Red triangles and blue squares are related to RXTE and ASCA observations, respectively.

betrween these correlations is in the BMC normalization values (proportional to $M_{\mathrm{BH}} / d^{2}$ ratio).

To estimate the BH mass, $M_{\mathrm{t}}$, of NGC 7469 (target source), one should slide the reference source correlation along the $N_{\mathrm{bmc}}-$ axis to that of the target source (see Fig. 10),

$M_{\mathrm{t}}=M_{\mathrm{r}} \frac{N_{\mathrm{t}}}{N_{\mathrm{r}}}\left(\frac{d_{\mathrm{t}}}{d_{\mathrm{r}}}\right)^{2} f_{\mathrm{G}}$,

where $t$ and $r$ correspond to the target and reference sources, respectively; a geometrical factor, $f_{G}=(\cos \theta)_{\mathrm{r}} /(\cos \theta)_{\mathrm{t}}$, the inclination angles $\theta_{\mathrm{r}}, \theta_{\mathrm{t}}$, and the distances $d_{\mathrm{r}}, d_{\mathrm{t}}$ are distances to the reference and target sources, correspondingly (see ST09). One can see values of $\theta$ in Table 6 but if some of these $\theta$-values are unavailable, then we assume that $f_{\mathrm{G}} \sim 1$.

In Fig. 10 we demonstrate the $\Gamma-N_{\text {bmc }}$ correlation for NGC 7469 (green points) obtained using the RXTE spectra along with the correlations for the two Galactic reference sources (GRO J1655-40 (pink), Cygnus X-1 (black)) and one extragalactic reference source NGC 4051 (blue line), which are similar to the correlations found for the target source. The BH masses and distances for each of these target-reference pairs are shown in Table 6.

After rearrangement, a BH mass, $M_{\mathrm{t}}$, for NGC 7469 can be evaluated using the formula (see TS16)

$M_{\mathrm{t}}=C_{0} N_{\mathrm{t}} d_{\mathrm{t}}^{2} f_{\mathrm{G}}$,

where $C_{0}=\left(1 / d_{\mathrm{r}}^{2}\right)\left(M_{\mathrm{r}} / N_{\mathrm{r}}\right)$ is the scaling coefficient for the reference source, $\mathrm{BH}$ masses $M_{\mathrm{t}}$ and $M_{\mathrm{r}}$ are in solar units, and $d_{\mathrm{r}}$ is the distance to a particular reference source measured in kiloparsecs.

We use values of $M_{\mathrm{r}}, M_{\mathrm{t}}, d_{\mathrm{r}}, d_{\mathrm{t}}$, and $\cos (i)$ from Table 6 and then we calculate the lowest limit of the mass, using the bestfit value of $N_{\mathrm{t}}=(1.25 \pm 0.04) \times 10^{-3}$ taken at the beginning of the index saturation (see Fig. 10) and measured in units of $L_{39} / d_{10}^{2} \mathrm{erg} \mathrm{s}^{-1} \mathrm{kpc}^{-2}$ (see Table 5 for values of the parameters of function $F\left(N_{\mathrm{t}}\right)$ Eq. (1)). Using $d_{\mathrm{r}}, M_{\mathrm{r}}, N_{\mathrm{r}}$ (see ST09), we found that $C_{0} \sim 2.0,1.9$, and 1.83 for NGC 4051, GRO J1555-40, and Cyg X-1. Finally, we obtain $M_{7469} \geq 3 \times 10^{6} M_{\odot}\left(M_{7469}=M_{\mathrm{t}}\right)$ assuming $d_{7469} \sim 70 \mathrm{Mpc}$ (Behar et al. 2017) and $f_{\mathrm{G}} \sim 1$. We summarize all these results in Table 6 ,

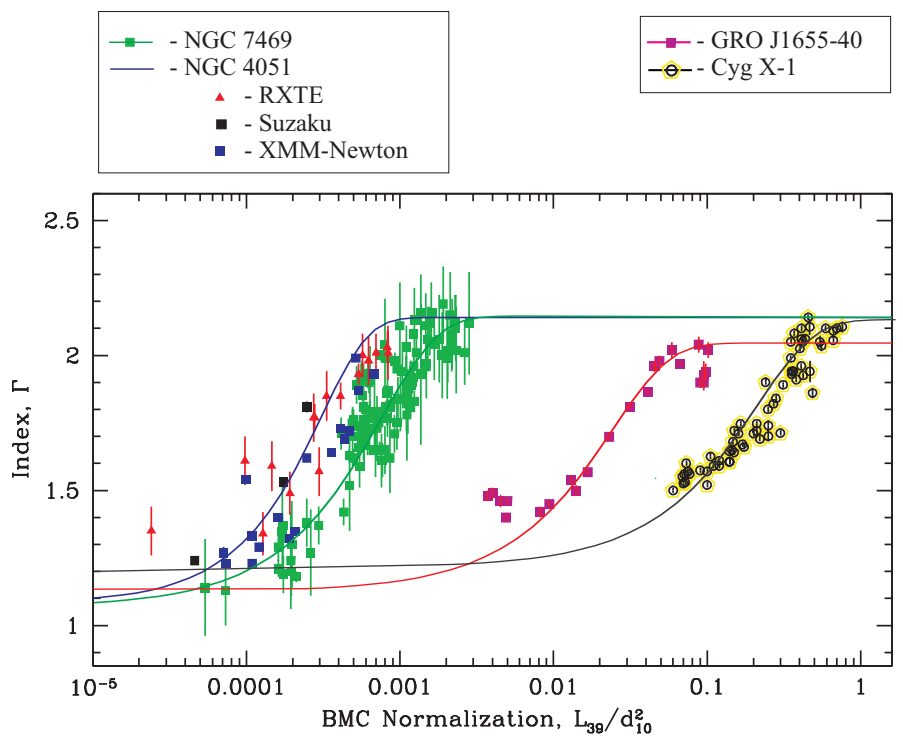

Fig. 10. Scaling of the photon index $\Gamma$ versus the normalization $N_{\mathrm{BMC}}$ for NGC 7469 (green line is the target source) as well as NGC 4051, GRO J1655-40, and Cygnus X-1 (reference sources). Red triangles stand for RXTE, black squares for $S u z a k u$, and blue squares for XMM-Newton data for NGC 4051. Pink squares and yellow-black circles correspond to RXTE data for GRO J1655-40 and Cygnus X-1, respectively.

The obtained $\mathrm{BH}$ mass estimate is in agreement with a high bolometric luminosity for NGC 7469 and $k T_{\mathrm{s}}$ value, which is in the range of $140-200 \mathrm{eV}$ using the ASCA spectra. For example, Shakura \& Sunyaev (1973; see also Novikov \& Thorne 1973) provide an effective temperature of the accretion material of $T_{\mathrm{eff}} \sim T_{\mathrm{s}} \propto M_{\mathrm{BH}}^{-1 / 4}$.

It is also important to emphasize that our NGC 7469 lower mass estimate is consistent with a SMBH mass of $(1-6) \times 10^{7} M_{\odot}$ (Peterson et al. 2004, 2014; Shapovalova \& Popovic 2017) estimated by the reverberation mapping method. The derived $\mathrm{BH}$ mass is the lower limit estimate only, because the photon index versus the normalization has an uncertainty with geometrical factor $f_{\mathrm{G}}=\left(\cos i_{\mathrm{d}}\right)_{\mathrm{r}} / /\left(\cos i_{\mathrm{d}}\right)_{\mathrm{t}}$. Generally, the photon index versus the QPO frequency correlation enables us to obtain the precise BH mass (see ST09), but QPOs are very hard to detect in the power spectra from AGNs. In the Introduction we emphasize that a BH mass estimate of NGC 7469 is in a fairly wide range. Although our BH mass estimate is only the lower limit of that, it significantly constrains the range of a BH mass for NGC 7469 (see Table 6).

We derived the bolometric luminosity using the normalization of the BMC model and obtain one between $2 \times 10^{43} \mathrm{erg} \mathrm{s}^{-1}$ and $6 \times 10^{44} \mathrm{erg} \mathrm{s}^{-1}$ (assuming isotropic radiation). Observations of many Galactic BHs (GBHs) and their X-ray spectral analysis (see ST09, Titarchuk \& Seifina 2009, Seifina \& Titarchuk 2010 and STS14, SCT18) confirm the TZ98 prediction that the spectral (photon) index saturates with the mass accretion rate, obviously related to the Eddington ratio. This value is not so far from the Eddington limit for the obtained BH mass and assumed source distance (see Table 6). For our particular source NGC 7469, we also reveal that the photon index, $\Gamma$, monotonically increases from 1.2 and then finally saturates at a value of 2.1 (see Fig. 9).

\section{Conclusions}

We found the low-intermediate state transitions observed in NGC 7469 using the full set of ASCA (1993-1994) and RXTE 
Table 5. Parameterizations for reference and target sources.

\begin{tabular}{lccccc}
\hline \hline Reference source & $\mathcal{A}$ & $\mathcal{B}$ & $\mathcal{D}$ & $x_{\text {tr }}$ & $\beta$ \\
\hline GRO J1655-40 & $2.03 \pm 0.02$ & $0.45 \pm 0.03$ & 1.0 & $0.07 \pm 0.02$ & $1.9 \pm 0.2$ \\
Cyg X-1 & $2.09 \pm 0.01$ & $0.52 \pm 0.02$ & 1.0 & $0.4 \pm 0.1$ & $3.5 \pm 0.1$ \\
NGC 4051 & $2.05 \pm 0.07$ & $0.61 \pm 0.08$ & 1.0 & {$[9.54 \pm 0.2] \times 10^{-4}$} & $0.52 \pm 0.09$ \\
\hline Target source & $\mathcal{A}$ & $\mathcal{B}$ & $\mathcal{D}$ & $x_{\text {tr }}\left[\times 10^{-3}\right]$ & $\beta$ \\
\hline NGC 7469 & $2.04 \pm 0.06$ & $0.62 \pm 0.03$ & 1.0 & $1.25 \pm 0.04$ & $0.62 \pm 0.04$ \\
\hline
\end{tabular}

Table 6. BH masses and distances.

\begin{tabular}{|c|c|c|c|c|}
\hline Source & $M_{\text {dyn }}\left(M_{\odot}\right)$ & $i_{\text {orb }}(\operatorname{deg})$ & $d(\mathrm{kpc})$ & $M_{\text {scal }}\left(M_{\odot}\right)$ \\
\hline GRO J1655-40 & $6.3 \pm 0.3^{(1,2)}$ & $70 \pm 1^{(1,2)}$ & $3.2 \pm 0.2^{(3)}$ & \\
\hline Cyg X-1 & $6.8-13.3^{(4,5)}$ & $35 \pm 5^{(4,5)}$ & $2.5 \pm 0.3^{(4,5)}$ & $7.9 \pm 1.0$ \\
\hline NGC $4051^{(6,7,8,9,10)}$ & $\ldots$ & $\ldots$ & $\sim 10 \times 10^{3}$ & $\geq 6 \times 10^{5}$ \\
\hline NGC $7469^{(11,12,13)}$ & $\ldots$ & $\ldots$ & $\sim 70 \times 10^{3}$ & $\geq 3 \times 10^{6}$ \\
\hline
\end{tabular}

References. (1) Greene et al. (2001); (2) Hjellming \& Rupen (1995); (3) Jonker \& Nelemans (2004); (4) Herrero (1995); (5) Ninkov et al. (1987); (6) MHardy et al. (2004); (7) Haba et al. (2008); (8) Pounds \& King (2013); (9) Lobban et al. (2011); (10) Terashima et al. (2009); (11) Peterson et al. (2004); (12) Peterson et al. (2014); and (13) Shapovalova \& Popovic (2017).

observations (1996-2009) and we demonstrated the validity of fitting the observed spectra applying the BMC model for all observations, independent of the spectral state of the source. We investigated the X-ray outburst properties of NGC 7469 and confirmed the presence of spectral state transitions during the outbursts using the index-normalization (or $\dot{M}$ ) correlation observed in NGC 7469, which were similar to those in Galactic BHs as well as to a number of extragalactic BH sources. In particular, we found that NGC 7469 follows the $\Gamma-\dot{M}$ correlation previously obtained for an extragalactic $\mathrm{SMBH}$ source, NGC 4051 (SST18), and for the Galactic BHs GRO J1655-40 and Cyg X-1 (ST09), for which we take account of the particular values of the $M_{\mathrm{BH}} / d^{2}$ ratio (see Fig. 10).

The photon index $\Gamma$ of the NGC 7469 spectra is in the range of $\Gamma=1.2-2.1$.

We tested a possible effect of the presence of the narrow line region (NLR) on our results and conclude that the NLR contribution in the spectrum is quite low in the case of NGC 7469.

We also find that the peak bolometric luminosity is about $4 \times 10^{44} \mathrm{erg} \mathrm{s}^{-1}$. We used the observed index-mass accretion rate correlation to estimate $M_{\mathrm{BH}}$ in NGC 7469. This scaling method was successfully applied to find BH masses of Galactic (e.g., ST09, STS13) and extragalactic black holes (TS16; Sobolewska \& Papadakis 2009; Giacche et al. 2014; Titarchuk \& Seifina 2017; Seifina et al. 2017, 2018). An application of the scaling technique to the X-ray data from ASCA and RXTE observations of NGC 7469 allows us to estimate $M_{\mathrm{BH}}$ for this particular source. We found values of $M_{\mathrm{BH}} \geq 3 \times 10^{6} M_{\odot}$.

Furthermore, our BH mass estimate is in agreement with the previous $\mathrm{BH}$ mass evaluations of more than $10^{6} M_{\odot}$ derived using detailed X-ray spectral modeling (Peterson et al. 2004, 2014; Shapovalova \& Popovic 2017). Combining all these estimates with the inferred low temperatures of the seed disk photons $k T_{\mathrm{s}}$, we establish that the compact object of NGC 7469 is likely to be a SMBH with at least $M_{\mathrm{BH}}>3 \times 10^{6} M_{\odot}$.

Acknowledgements. We acknowledge the interesting remarks and points of the referee.

\section{References}

Alloin, D., Clavel, J., \& Peterson, B. M. 1994, in Frontiers of Space and Ground-based Astronomy, eds. W. Wamsteker, M. S. Longair, \& Y. Kondo (Dordrecht: Kluwer), 423

Artamonov, B. P., Bruevich, V. V., Gusev, A. S., et al. 2010, Astron. Rep., 54, 767

Barr, P. 1986, MNRAS, 436, L35

Behar, E., Peretz, U., Kriss, G. A., et al. 2017, A\&A, 601, A17

Bottinelli, L., Gouguenheim, L., Paturel, G., \& de Vaucouleurs, G. 1984, A\&AS, 56,381

Bradt, H. V., Rothschild, R. E., \& Swank, J. H. 1993, A\&AS, 97, 533

Chesnok, N. G., Sergeev, S. G., \& Vavilova, I. B. 2009, Kinematics Phys. Celestrial Bodies, 97, 533

Doroshenko, V. T., Lyuty, V. M., \& Rakhimov, V. Yu. 1989, Pis'ma Astron. Zh., 15,483

Doroshenko, V. T., Lyuty, V. M., \& Shenavrin, V. I. 1998, Astron. Lett., 24, 160

Doroshenko, V. T., Sergeev, S. G., Vovk, E. Yu., et al. 2010, Astron. Lett., 36, 611

Dultzin-Hacyan, D., Shuster, W. J., Parrao, L., et al. 1992, AJ, 103, 1796

Dultzin-Hacyan, D., Ruelas-Mayorga, A., \& Costero, R. 1993, Rev. Mex. Astron. Astrofis., 25, 143

Forman, W., Jones, C., Cominsky, L., et al. 1978, ApJS, 38, 357

Ganeshalingam, M., Li, W., \& Filippenko, A. V. 2013, MNRAS, 433, 2240

Gaskell, C. M. 2006, Proc. ASP Conf. Ser., eds. Gaskell, C. M., McHandy, I. M., Peterson, B. M., \& Sergeev, S. G., 360, 111

Gaskell, C. M. 2007, Proc. ASP Conf. Ser., eds. Ho, L. C., \& Wang, J.-M., 373, 596

Gehrels, N., Chincarini, G., Giommi, P., et al. 2004, ApJ, 611, 1005

George, I. M., \& Fabian, A. C. 1991, MNRAS, 249, 352

Giacche, S., Gili, R., \& Titarchuk, L. 2014, A\&A, 562, A44

Greene, J., Bailyn, C. D., \& Orosz, J. A. 2001, ApJ, 554, 1290

Guinazzi, M., Matsuoa, M., Piro, L., et al. 1994, ApJ, 436, L35

Haba, Y., Liebmann, A. C., \& Fukumura, K. 2008, Publ. Astron. Soc. Japan, 60, 1257

Herrero, J., Kudritzki, R. P., Gabler, R., Vilchez, J. M., \& Gabler, A. 1995, A\&A, 297, 556

Hjellming, R. M., \& Rupen, M. P., 1995, Nature, 375, 464

Jonker, P. G., \& Nelemans, G. 2004, MNRAS, 354, 355

Koss, M., Romero-Cañizares, C., Baronchelli, L., et al. 2015, ApJ, 807, 149

Laming, J. M., \& Titarchuk, L. 2004, ApJ, 615, L121

Laurent, P., \& Titarchuk, L. 1999, ApJ, 511, 289 (LT99)

Laurent, P., \& Titarchuk, L. 2011, ApJ, 727, L34

Liang, E. W., \& Liu, H. T. 2003, MNRAS, 340, L632

Lobban, A. P., Reeves, J. N., \& Miller, L. 2011, MNRAS, 414, 1965

Lyuty, V. M. 2005, Astron. Lett., 31, 645

Lyuty, V. M., \& Doroshenko, V. T., 1999, Astron. Lett., 25, 341 
Lyutyi, V. M., Doroshenko, V. T., Metlov, V. G., \& Irsmambetova, T. R. 1995, Astron. Lett., 21, 581

Matt, G., Perola, G. C., \& Piro, L. 1991, A\&A, 247, 25

$M^{\mathrm{c}}$ Hardy, I. M., Papadakis, I. E., Uttley, P., et al. 2004, MNRAS, 348, 783

Merkulova, N. I. 2000, AJ, 119, 631

Nandra, K., \& George, I. M. 1991, MNRAS, 267, 947

Nandra, K., Pounds, K. A., Stewart, G. C., et al. 1991, MNRAS, 248, 760

Netzer, H., \& Peterson, B. M., 1997, in Astronomical Time Series, eds. D. Maoz, A. Sternberg, \& E. M. Leibowitz (Dordrecht: Kluwer), 85

Ninkov, Z., Walker, G. A. H., \& Yang, S. 1987, ApJ, 321, 425

Novikov, I. D., \& Thorne, K. S. 1973, Black holes, eds. Dewitt, C., \& Dewitt, B. (N. Y.: Gorden \& Breach), 343

O’Brien, P. T., \& Leighly, K. M. 1998, Adv. Space Res., 21, 67

Orosz, J. A., et al. 2002, ApJ, 568, 84

Paggi, A., Wang, J., Fabbiano, G., \& Karovska, M. 2012, ApJ, 756, 39

Peterson, B. M., Ferrarese, L., Gilbert, K. M., et al. 2004, ApJ, 613, 682

Peterson, B. M., Grier, C. J., Horne, Keith, Pogge, R. W., et al. 2014, ApJ, 795, 149

Piro, L., Yamauchi, M., \& Matsuoka, M. 1990, ApJ, 360, L35

Pounds, K. A., \& King, A. R. 2013, MNRAS, 433, 1369

Rees, M. 1988, Nature, 333, 523

Rivers, E., Markowitz, A., \& Rothschild, R. 2013, ApJ, 772, 114

Scott, E., Kriss, G. A., Lee, J. C., et al. 2005, ApJ, 634, 193

Seifina, E., \& Titarchuk, L. 2010, ApJ, 722, 586 (ST10)

Seifina, E., \& Titarchuk, L. 2011, ApJ, 738, 128 (ST11)

Seifina, E., \& Titarchuk, L. 2012, ApJ, 747, 99 (ST12)

Seifina, E., Titarchuk, L., \& Frontera, F. 2013, ApJ, 766, 63 (STF13)

Seifina, E., Titarchuk, L., \& Shaposhnikov, N. 2014, ApJ, 789, 57 (STS14)

Seifina, E., Titarchuk, L., Shrader, Ch., \& Shaposhnikov, N. 2015, ApJ, 808, 142 (STSS15)

Seifina, E., Titarchuk, L., \& Virgilli, E. 2017, A\&A, 607, A38

Seifina, E., Chekhtmann, A., \& Titarchuk, L. 2018, A\&A, 613, A48 (SCT18)

Sergeev, S. G., Doroshenko, V. T., Golubinskiy, Yu. V., et al. 2005, ApJ, 622, 129
Seyfert, C. K. 1943, ApJ 97, 28

Shakura, N. I., \& Sunyaev, R. A. 1973, A\&A, 24, 337

Shaposhnikov, N., \& Titarchuk, L. 2004, ApJ, 606, L57

Shaposhnikov, N., \& Titarchuk, L. 2006, ApJ, 643, 1098 (ST06)

Shaposhnikov, N., \& Titarchuk, L. 2007, ApJ, 663, 445

Shaposhnikov, N., \& Titarchuk, L. 2009, ApJ, 699, 453 (ST09)

Shaposhnikov, N., Swank, J., Shrader, C. R., et al. 2007, ApJ, 655, 434

Shapovalova, A. I., Popovic, L. C., et al. 2017, MNRAS, 466, 4759

Sobolewska, M. A., \& Papadakis, I. E. 2009, MNRAS, 399, 1997

Spirandob, C. M., Haynes, M. P., Giovanelli, R., et al. 2005, ApJS, 160, 149

Suganuma, M., Yoshii, Y., Kobayashi, Y., et al. 2006, ApJ, 639, 46

Tanaka, Y., Inoue, H., \& Holt, S. S. 1994, PASJ, 46, L37

Terashima, Y., Gallo, L. C., \& Inoue, H. 2009, PASJ, 61, S299

Theureau, G., Hanski, M. O., Coudreau, N., Hallet, N., \& Martin, J. M. 2007, A\&A, 465,71

Titarchuk, L. G., \& Fiorito, R. 2004, ApJ, 612, 988 (TF04)

Titarchuk, L., \& Seifina, E. 2009, ApJ, 706, 1463

Titarchuk, L., \& Seifina, E. 2016, A\&A, 585, A94 (TS16)

Titarchuk, L., \& Seifina, E. 2017, A\&A, 602, A113 (TS17)

Titarchuk, L., \& Shaposhnikov, N. 2005, ApJ, 626, 298

Titarchuk, L., \& Zannias, T. 1998, ApJ, 499, 315 (TZ98)

Titarchuk, L., Mastichiadis, A., \& Kylafis, N. D. 1997, ApJ, 487, 834

Titarchuk, L., Lapidus, I. I., \& Muslimov, A. 1998, ApJ, 499, 315 (TLM98)

Turner, T. J., Weaver, K. A., Mushshotzky, R. F., Holt, S. S., \& Madejski, G. M. 1991, ApJ, 381, 85

Turner, T. J., Weaver, K. A., \& Mushshotzky, R. F. 1993, ApJ, 412, 72

Ugolkova, L. S., \& Artamonov, B. P. 2011, Odessa Astron. Publ., 24, 78

Ugolkova, L. S., Artamonov, B. P., Shimanovskaya, E. V., et al. 2017, Astron. Lett., 43,233

Ulrich, M.-H., Maraschi, L., \& Urry, C. M. 1997, ARA\&A, 35, 445

Wakker, B. P., Lockman, F. J., \& Brown, J. M. 2011, ApJ, 728, 159

Wang, J.-M., Du, P., Hu, Ch, Netzer, H., Bai, J.-M., et al. 2014, ApJ, 793, 108 